\title{
The Quantum Structure of Spacetime at the Planck Scale and Quantum Fields
}

\author{
Sergio Doplicher ${ }^{1}$, Klaus Fredenhagen $^{2}$, John E. Roberts $^{3}$ \\ ${ }^{1}$ Dipartimento di Matematica, Università di Roma "La Sapienza", I-00185 Roma, Italy \\ 2 II Institut für Theoretische Physik der Universität Hamburg, D-22761 Hamburg, Germany \\ ${ }^{3}$ Dipartimento di Matematica, Università di Roma "Tor Vergata", I-00133 Roma, Italy \\ 1,3 Research supported by MRST and CNR-GNAFA
}

Commun. Math. Phys. 172, 187-220 (1995)

Received: 22 June 1994

\begin{abstract}
We propose uncertainty relations for the different coordinates of spacetime events, motivated by Heisenberg's principle and by Einstein's theory of classical gravity. A model of Quantum Spacetime is then discussed where the commutation relations exactly implement our uncertainty relations.

We outline the definition of free fields and interactions over QST and take the first steps to adapting the usual perturbation theory. The quantum nature of the underlying spacetime replaces a local interaction by a specific nonlocal effective interaction in the ordinary Minkowski space. A detailed study of interacting QFT and of the smoothing of ultraviolet divergences is deferred to a subsequent paper.

In the classical limit where the Planck length goes to zero, our Quantum Spacetime reduces to the ordinary Minkowski space times a two component space whose components are homeomorphic to the tangent bundle $T S^{2}$ of the 2 -sphere. The relations with Connes' theory of the standard model will be studied elsewhere.
\end{abstract}

\section{Introduction}

It is generally believed that the picture of spacetime as a manifold $M$ locally modelled on the flat Minkowski space $M_{0}=\mathbb{R}^{4}$ should break down at very short distances of the order of the Planck length

$$
\lambda_{P}=\left(\frac{G \hbar}{c^{3}}\right)^{1 / 2} \simeq 1.6 \times 10^{-33} \mathrm{~cm} .
$$

Limitations in the possible accuracy of localization of spacetime events should in fact be a feature of a Quantum Theory incorporating gravitation.

There have been investigations on possible mechanisms leading to such limitations in the context of string theory [1,2], in Ashtekar's approach to quantum gravity [3], and, in a more formal way, in the context of Quantum Groups [4,5]. 
These different approaches have led to different limitations and, more significantly, to different pictures of spacetime where gravitational effects in the small are necessarily strong (spacetime foam [6]).

Our proposal differs radically: attempts to localize with extreme precision cause gravitational collapse so that spacetime below the Planck scale has no operational meaning. We elaborate on this well known remark and are led to spacetime uncertainty relations. In our proposal, spacetime has a quantum structure intrinsically implying those relations. Thus the impossibility of giving an operational meaning to spacetime in the small is incorporated in the mathematical structure of the model.

Similar models can be found in the work of J. Madore [7], where, however, no attempt was made to motivate the algebraic structure by an operational analysis of localization.

We thus propose that spacetime ought to be described as a non-commutative manifold, i.e. the commutative algebra $\mathcal{C}_{0}(M)$ of complex continuous functions on $M$ vanishing at infinity should be replaced by a non-commutative algebra $\mathcal{E}$, and points of $M$ by pure states on $\mathcal{E}$. The aim of this paper is to propose an algebra $\mathcal{E}$ describing Quantum Spacetime and to look for a formulation of QFT over QST.

We now formulate some criteria for the choice of $\mathcal{E}$. We are interested in elementary particle physics, that is in describing idealized situations where only few colliding particles are present. Therefore, as a first state of our project, we are interested in a non-commutative variant of the flat spacetime $M_{0}$, deviating from $M_{0}$ only at very short distances, which fulfils the following principles:

1) The commutation relations in $\mathcal{E}$ should be motivated by operationally meaningful uncertainty relations between the different coordinates of spacetime events.

2) The flat spacetime $M_{0}$ should appear (possibly as a factor) in the large scale limit of $\mathcal{E}$.

3) The full Poincaré group should act as symmetries on $\mathcal{E}$.

Condition 3 is motivated and made possible by 2) and allows us to adopt Wigner's description of elementary particles in terms of irreducible representations of the Poincaré group. Parity or time reversal symmetry breaking might be features of specific interactions but the quantum spacetime should be, as ordinary spacetime, reflection symmetric. Therefore, in 3) the full Poincaré group is required to yield symmetries of the quantum spacetime.

In Sect. 2 we explore the limitations of localization measurements which are due to the possible creation of black holes by concentration of energy.

We find the uncertainty relations

$$
\begin{gathered}
\Delta x_{0}\left(\Delta x_{1}+\Delta x_{2}+\Delta x_{3}\right) \gtrsim \lambda_{P}^{2}, \\
\Delta x_{1} \Delta x_{2}+\Delta x_{2} \Delta x_{3}+\Delta x_{3} \Delta x_{1} \gtrsim \lambda_{P}^{2},
\end{gathered}
$$

which are implied by those limitations but do not necessarily imply them. In Sect. 3 we find algebraic relations which imply these uncertainty relations. They have 
the form

$$
\begin{aligned}
q_{\mu} & =q_{\mu}^{*}, \\
{\left[\left[q_{\mu}, q_{\nu}\right], q_{\rho}\right] } & =0, \\
{\left[q_{\mu}, q_{\nu}\right]\left[q^{\mu}, q^{\nu}\right] } & =0, \\
\left(\frac{1}{8}\left[q_{\mu}, q_{\nu}\right]\left[q_{\rho}, q_{\sigma}\right] \varepsilon^{\mu \nu \rho \sigma}\right)^{2} & =\lambda_{P}^{8} .
\end{aligned}
$$

The resulting algebra has a centre generated by the commutators $i Q_{\mu \nu}=\left[q_{\mu}, q_{\nu}\right]$. The physical meaning of this centre has still to be understood. It is responsible for the fact that in the large scale limit, performed at fixed spectral values of the centre, the resulting classical space is $M_{0} \times \Sigma$, where

$$
\begin{aligned}
\Sigma & =\left\{\left(\sigma_{\mu \nu}\right), \sigma_{\mu \nu}=-\sigma_{\nu \mu}, \sigma_{\mu \nu} \sigma^{\mu \nu}=0,\left(1 / 8 \sigma_{\mu \nu} \sigma_{\rho \sigma} \varepsilon^{\mu \nu \rho \sigma}\right)^{2}=1\right\} \\
& \simeq T S^{2} \times\{1,-1\}
\end{aligned}
$$

In Sect. 4 we define a $C^{*}$-algebra $\mathcal{E}$ to which the operators $q_{\mu}$ are affiliated. $\mathcal{E}$ turns out to be isomorphic to the algebra $C_{0}(\Sigma, \mathcal{K})$ of continuous functions from $\Sigma$ to $\mathcal{K}$ vanishing at infinity, where $\mathcal{K}$ is the algebra of compact operators in a fixed separable Hilbert space. States on $\mathcal{E}$ describe the possible localization of events. For optimal localization in a specific Lorentz frame, in the sense that $\Sigma\left(\Delta q_{\mu}\right)^{2}$ is minimal, the state must be concentrated on spectral values of the centre in a compact submanifold $\Sigma^{(1)}$ of $\Sigma$ with $\Sigma^{(1)} \simeq S^{2} \times\{1,-1\}$.

In Sect. 5 we develop calculus on $\mathcal{E}$. In particular we define a spacetime integral as a positive Poincaré invariant trace and integrals over spatial hyperplanes as positive weights. The existence of these latter integrals will be crucial for introducing an interaction and depends on the fact that the uncertainty relations admit an absolutely precise determination of time at the cost of complete uncertainty in at least one spatial coordinate.

In Sect. 6 we take the first steps towards quantum field theory on the quantum spacetime. We define free fields and show that their commutator at spacelike distances decreases like a Gaussian. We give a formal recipe for defining interaction Hamiltonians, interacting fields and the perturbative expansion of the $S$-matrix. This expansion could be derived from a specific nonlocal effective interaction on ordinary Minkowski space where the nonlocal corrections are at least quadratic in $\lambda_{P}$.

Gauge theories on the quantum spacetime should be formulated in the framework of non-commutative geometry [8]. More substantial deviations from theories on classical spacetime are to be expected; quantum electrodynamics, for example, will be a non-Abelian gauge theory. The occurrence of the two point set $\{1,-1\}$ in the classical limit recalls Connes' theory of the standard model $[8,9]$. We hope to take up these problems elsewhere.

The quantum aspects of gravitation might, however, well lead to a more drastic deviation from the classical structure of spacetime than is shown in our model, which is motivated by semiclassical arguments pertaining to classical gravity.

Some of the structures discussed in this paper have already appeared in the literature in different contexts. Thus our commutation relations occur in the theory of charged particles is constant electromagnetic fields (see e.g. [10]). A Euclidean version of fields on a non-commutative spacetime related to ours was proposed in 
[11]. The Schwinger model on Madore's fuzzy sphere [7] was discussed in [12]. For other models of quantum spacetime see e.g. [13].

A less technical version of our main results has appeared in [14].

\section{Spacetime Uncertainties}

We start our discussion by pointing out that combining Heisenberg's uncertainty principle with Einstein's theory of classical gravity leads to the conclusion that ordinary spacetime loses any operational meaning in the small.

Measuring a spacetime coordinate with great accuracy $a$ causes an uncertainty in momentum of the order $\frac{1}{a}$ (unless otherwise stated, we will use natural units $\hbar=c=G=1$ ). Neglecting rest masses, an energy of the order $\frac{1}{a}$ is transmitted to the system and concentrated at some time in the localization region. The associated energy-momentum tensor $T_{\mu \nu}$ generates a gravitational field which, in principle, should be determined by solving Einstein's equations for the metric $\eta_{\mu \nu}$,

$$
R_{\mu \nu}-\frac{1}{2} R \eta_{\mu \nu}=8 \pi T_{\mu \nu}
$$

The smaller the uncertainties $\Delta x_{\mu}$ in the measurement of coordinates, the stronger will be the gravitational field generated by the measurement. When this field becomes so strong as to prevent light or other signals from leaving the region in question, an operational meaning can no longer be attached to the localization.

Our task is now to investigate how localization is restricted by requiring that no black hole is produced in the course of measurement. Since pair creation is important in processes involving high energy transfer, the framework of quantum field theory has to be used.

Our information on the localization of events in spacetime is obtained by using operations which prepare a state localized in a region with sides of lengths $\Delta x_{\mu}$, that we take as a measure of the uncertainties in the spacetime coordinates of an event. We will admit only those states whose associated energy-momentum tensor, taken as a source in Einstein's equation, does not generate closed trapped surfaces in the sense of Penrose ${ }^{1}$.

As a consequence, the $\Delta x_{\mu}$ will be subject to some restrictions preventing them from being simultaneously arbitrarily small. We may then pose our

Uncertainty Problem: Find the restrictions on the uncertainties $\Delta x_{0}, \ldots, \Delta x_{3}$ valid in all admissible states.

To formulate our problem precisely, we turn to the free neutral scalar field and use the coherent states as a model for a class of states prepared by such operations. The corresponding state vector have the form

$$
\Phi=e^{i \varphi(f)} \Omega,
$$

where $\Omega$ denotes the vacuum state vector and $f$ a real smooth test function with compact support where the state $(2.2)$ is strictly localized $[16,17]$. We have to choose the function $f$ so that $\Phi$ differs significantly from the vacuum in the support of $f$, a region whose extent is characterized by $\Delta x_{\mu}, \mu=0, \ldots, 3$. To this end we

\footnotetext{
${ }^{1}$ Cf. $[15,8.2]$ : we are grateful to D. Boccaletti for calling our attention to this reference.
} 
require the state to be significantly different from its translate through $\Delta x_{\mu}$. This may be expressed by the condition

$$
\left|\left(\Phi, P_{\mu} \Phi\right)\right| \geq\left(\Delta x_{\mu}\right)^{-1}
$$

which will be analyzed more thoroughly elsewhere.

The mean energy-momentum tensor of the state induced by (2.2) is given by

$$
t_{\mu \nu}(x ; f)=\left(\Phi,: T_{\mu \nu}(x): \Phi\right)
$$

where : $T_{\mu \nu}$ : is the normal form of the energy-momentum density of the field

$$
\begin{gathered}
T_{\mu \nu}(x)=\frac{\partial \varphi}{\partial x^{\mu}}(x) \frac{\partial \varphi}{\partial x^{\nu}}(x)+\frac{1}{2}\left(m^{2} \varphi(x)^{2}-\frac{\partial \varphi}{\partial x^{\lambda}}(x) \frac{\partial \varphi}{\partial x_{\lambda}}(x)\right) g_{\mu \nu} \\
: T_{\mu \nu}(x):=T_{\mu \nu}(x)-\left(\Omega, T_{\mu \nu}(x) \Omega\right) .
\end{gathered}
$$

The mean energy-momentum tensor (2.3) coincides with the energy-momentum density associated with a suitable solution $\psi_{f}$ of the Klein-Gordon equation, $(\square+$ $\left.m^{2}\right) \psi_{f}=0$, using the expression (2.4) in classical field theory. Here

$$
\begin{aligned}
\psi_{f}(x) & =\operatorname{Im} \int_{\Omega_{m}^{+}} e^{-i k x} \hat{f}(k) d \Omega_{m}(k) \\
& =\int \Delta_{m}(x-y) f(y) d^{4} y
\end{aligned}
$$

(where $d \Omega_{m}$ is the invariant measure on the positive energy mass $m$ hyperboloid $\Omega_{m}^{+}$and $\Delta_{m}$ the commutator function for the free scalar field of mass $m$ ). By the support properties of $\Delta_{m}, \psi_{f}$ is localized at some time with the same accuracy $\Delta x_{1}$, $\Delta x_{2}, \Delta x_{3}$ as $\Phi$; furthermore, by $(2.6), \psi_{f}$ cannot be a positive energy solution since $f$ has compact support. However, $\psi_{f}$ has the same mean energy

$$
\left(\psi_{f}, h \psi_{f}\right)=(\Phi, H \Phi)
$$

where $h$ is the one particle Hamiltonian and $H$ the free field Hamiltonian. Thus our problem is equivalent to its variant dealing only with wave functions. We will not try to solve this problem here; instead we propose as an ansatz the following spacetime uncertainty relations in generic units:

$$
\begin{gathered}
\Delta x_{0} \sum_{j=1}^{3} \Delta x_{j} \gtrsim \lambda_{P}^{2} \\
\sum_{j<k=1}^{3} \Delta x_{j} \Delta x_{k} \gtrsim \lambda_{P}^{2} .
\end{gathered}
$$

We will motivate these relations heuristically limiting ourselves to a crude estimate, where (2.1) is replaced by the linearized equations, the components of $T_{\mu \nu}$ with $(\mu, \nu) \neq(0,0)$ are neglected, and the total energy $E$, where $E \sim \frac{1}{\Delta x_{0}}$ if $\Delta x_{0}$ is very 
small, is supposed to be distributed with constant density $\rho(-t)$ at negative times $x_{0}=-t$ over a volume with sides $\Delta x_{1}+t, \Delta x_{2}+t, \Delta x_{3}+t$.

In other words we assume uniform spreading with all speeds not exceeding the speed of light and localization around the origin, up to $\Delta x_{1}, \Delta x_{2}, \Delta x_{3}$, at $x_{0}=0$.

The gravitational potential $\varphi$ at $x_{\mu} \cong 0$ can be evaluated as the retarded potential

$$
\varphi \sim-\int \frac{\varphi(\vec{y},-r)}{r} d^{3} \vec{y} \sim-\frac{1}{\Delta x_{0}} \int_{0}^{\infty} \frac{1}{r} \frac{r^{2} d r}{\left(\Delta x_{1}+r\right)\left(\Delta x_{2}+r\right)\left(\Delta x_{3}+r\right)},
$$

and we impose the condition that photons of energy $\varepsilon$ should not be trapped, i.e. $\varepsilon+\varepsilon \varphi \gtrsim 0$ or $-\varphi \lesssim 1$.

It is easy to compute the leading behaviour of (2.10) in the three regimes: $\Delta x_{1} \sim$ $\Delta x_{2} \sim \Delta x_{3} ; \Delta x_{1} \sim \Delta x_{2} \gg \Delta x_{3} ; \Delta x_{1} \gg \Delta x_{2} \sim \Delta x_{3}$. One finds $\Delta x_{0} \cdot \Delta x_{1} \gtrsim 1$ in the first two cases, and $\Delta x_{0} \cdot \Delta x_{1} \gtrsim \ln \frac{\Delta x_{1}}{\Delta x_{2}}$ in the third. If $\Delta x_{1} / \Delta x_{2}$ is of the order of unity we are back to the first case, so that $\Delta x_{0} \cdot \Delta x_{1} \gtrsim 1$ is the absolute limitation, according to (2.8), in the third case too. If $\Delta x_{j} \ll \Delta x_{0}$ we must take $E \sim \frac{1}{\Delta x_{j}}$ and we find (2.9).

If $\Delta x_{0}$ is very large compared with $\Delta x_{j}, j=1,2,3$, we can take as an extreme idealization a static solution and the Schwarzschild and Kerr solutions motivate (2.9). For, if $\Delta x_{1} \sim \Delta x_{2} \sim \Delta x_{3} \sim a$, we could take a spherically symmetric solution with mass $\frac{1}{a}$, and our condition says that $a$ should be not smaller than the Schwarzschild radius $\sim \frac{1}{a}$, i.e. $a \gtrsim 1$.

If, say $\Delta x_{1} \sim \Delta x_{2} \sim r \gg \Delta x_{3} \sim a$, we may take the axially symmetric Kerr solution with mass $M \sim \frac{1}{a}$ and angular momentum $L$; the limit case is $L / M \sim M$ and $L \lesssim M r[18$, Ch. 7$]$ which gives

$$
a \cdot r \gtrsim 1
$$

in accordance with (2.9). We might further argue that, in the extreme situation considered, the energy density is actually concentrated on a thin ring region of radius $r$ and thickness $a$, so that the requirement expressed by (2.9) that at least one space uncertainty is large is not in contradiction with the Kerr solution.

However condition (2.9) is actually weaker since it allows $\Delta x_{1} \sim a$ to be arbitrarily small and $\Delta x_{2} \sim \Delta x_{3} \sim r$ to be of order one.

In the next section we discuss covariant commutation relations which do imply that the uncertainty relations (2.8) (2.9) hold in each state over the associated algebra. We interpret these states as describing possible localizations of measurements.

The separation of the spacetime localization from the measurement of a local observable is due to our classical treatment of the gravitational effects of localization. The present approach ought to be considered as a semiclassical approximation to a theory, presently unknown, where gravity and quantum physics are truly unified. 


\section{Quantum Conditions on Minkowski Space}

Let $A_{1}, A_{2}, \ldots, A_{n}$ be elements of a complex algebra; their non-commutativity can be measured by the quantity

$$
\begin{aligned}
{\left[A_{1}, \ldots, A_{n}\right] } & \equiv \sum \varepsilon_{i_{1} \ldots i_{n}} A_{i_{1}} \ldots A_{i_{n}} \\
& =\operatorname{det}\left(\begin{array}{c}
A_{1} \ldots A_{n} \\
A_{1} \ldots A_{n} \\
\ldots \\
A_{1} \ldots A_{n}
\end{array}\right) .
\end{aligned}
$$

If $n=2$, this is just the commutator; if $n=3$ and for $(j, l, k)=(1,2,3)$ and cyclic permutations we set

$$
\left[A_{j}, A_{l}\right]=i C_{k}
$$

then

$$
\left[A_{1}, A_{2}, A_{3}\right]=i \sum_{k} A_{k} C_{k} .
$$

If $n=4$, we think of operators $q_{0}, \ldots, q_{3}$ describing the coordinates in Quantum Spacetime and forming a Lorentz vector and we will use covariant notation. Define

$$
i Q_{\mu \nu}=\left[q_{\mu}, q_{\nu}\right], \quad \mu, \nu=0,1,2,3 .
$$

Antisymmetry of $\varepsilon$ then gives

$$
\left[q_{0}, q_{1}, q_{2}, q_{3}\right]=\varepsilon^{\mu \nu \lambda \rho} q_{\mu} q_{\nu} q_{\lambda} q_{\rho}=-\frac{1}{4} \varepsilon^{\mu \nu \lambda \rho} Q_{\mu \nu} Q_{\lambda \rho},
$$

so that, setting

$$
(* Q)^{\mu \nu}=\frac{1}{2} \varepsilon^{\mu \nu \lambda \rho} Q_{\lambda \rho}
$$

as usual, we get

$$
\left[q_{0}, \ldots, q_{3}\right]=-\frac{1}{2} Q_{\mu \nu}(* Q)^{\mu \nu} .
$$

If we denote by $\vec{e}, \vec{m}$ the "electric" and "magnetic" components of $Q$, respectively, i.e.

$$
\begin{aligned}
& Q_{0 j}=e_{j}=(* Q)^{l k} \\
& Q_{l k}=m_{j}=(* Q)^{0 j}
\end{aligned}
$$

the two independent Lorentz invariants which can be constructed with the tensor $Q_{\mu \nu}$ are given by

$$
\begin{gathered}
\frac{1}{2} Q_{\mu \nu} Q^{\mu \nu}=\vec{m}^{2}-\vec{e}^{2} \\
\frac{1}{2} Q_{\mu \nu}(* Q)^{\mu \nu}=-\left[q_{0}, \ldots, q_{3}\right]=\vec{e} \cdot \vec{m}+\vec{m} \cdot \vec{e} .
\end{gathered}
$$

These expressions are invariant under Poincaré transformations

$$
q \rightarrow \Lambda q+a \cdot I
$$




$$
a \in \mathbb{R}^{4}, \quad \Lambda \in L_{+}^{\uparrow} ;
$$

and total reflections $q \rightarrow-q$, but (3.7) is not separately invariant under space or time reflections since the sign of (3.7) changes. Therefore, the natural conditions which are Lorentz invariant and symmetric in $\vec{e}$ and $\vec{m}$ are the following:

$$
\begin{gathered}
Q_{\mu \nu} Q^{\mu \nu}=0, \\
\frac{1}{4}\left[q_{0}, q_{1}, q_{2}, q_{3}\right]^{2}=I .
\end{gathered}
$$

From now on, $q_{0}, q_{1}, q_{2}, q_{3}$ will be always assumed to be selfadjoint operators acting on a Hilbert space (or affiliated to a $C^{*}$-algebra, cf. [19] or Appendix A), and the operators $-i\left[q_{\mu}, q_{\nu}\right]$ will be assumed to have selfadjoint closures $Q_{\mu \nu}$.

We will now show that the conditions (3.9) do yield the uncertainty relations (2.8), (2.9) provided the $Q_{\mu \nu}$ are central, i.e. commute ${ }^{2}$ with the $q_{\mu}$ 's

$$
\left[q_{\lambda}, Q_{\mu \nu}\right]=0 ; \quad \lambda, \mu, \nu=0, \ldots, 3 .
$$

We will call Eq. (3.9) and (3.10) the Quantum Conditions.

For each selfadjoint operator $A$ on $\mathcal{H}$ and unit vector $x \in \mathcal{H}$, we will say that the vector state $\omega=\omega_{x}$ is in the domain of $A$ if $x \in \mathcal{D}_{A}$, so that

$$
\omega\left(A^{2}\right) \equiv(A x, A x)<\infty .
$$

The uncertainty $\Delta_{\omega} A$ is defined by $\left(\Delta_{\omega} A\right)^{2}=\omega\left((A-\omega(A) \cdot I)^{2}\right)=\omega\left(A^{2}\right)-\omega(A)^{2}$ and $\omega$ is said to be definite on $A$ if and only if $\Delta_{\omega} A=0$, i.e. $x$ is an eigenvector of $A$. The same applies if $A$ is affiliated to a $C^{*}$-algebra $\mathfrak{A}$ and $\omega \in \mathcal{S}(\mathfrak{A})$ is in the domain of $A$ (cf. Appendix A).

3.1 Theorem. Let the four selfadjoint coordinate operators $q_{0}, \ldots, q_{3}$ fulfill the Quantum Conditions (3.9), (3.10). For each state $\omega$ in the domain of the $\left[q_{\mu}, q_{\nu}\right]$, we have

$$
\begin{gathered}
\Delta_{\omega} q_{0} \sum_{j=1}^{3} \Delta_{\omega} q_{j} \geq \frac{1}{2}, \\
\sum_{1 \leq j<k \leq 3} \Delta_{\omega} q_{j} \Delta_{\omega} q_{k} \geq \frac{1}{2} .
\end{gathered}
$$

The proof will follow easily from the following propositions.

3.2 Proposition. Let $q_{0}, \ldots, q_{3}$ fulfill (3.9) and $\omega$ be a state in the domain of the $\left[q_{\mu}, q_{\nu}\right]$ 's, which is definite on $\left[q_{0}, q_{1}, q_{2}, q_{3}\right]$. Then

$$
\Delta_{\omega} q_{0} \cdot \sum_{j=1}^{3} \Delta_{\omega} q_{j}+\frac{1}{2} \sum_{j=1}^{3} \Delta_{\omega} e_{j} \geq \frac{1}{2},
$$

\footnotetext{
${ }^{2}$ Throughout this paper two selfadjoint operators will be said to commute if they commute strongly, i.e. their continuous functions vanishing at infinity (or, equivalently, their spectral resolutions) commute.
} 


$$
\sum_{1 \leq j<k \leq 3} \Delta_{\omega} q_{j} \Delta_{\omega} q_{k}+\frac{1}{2} \sum_{j=1}^{3} \Delta_{\omega} m_{j} \geq \frac{1}{2} .
$$

3.3 Proposition. Let $\mathfrak{A}$ be a $C^{*}$-algebra with unit $I$ and $\nu$ a (regular) probability measure on the state space $\mathcal{S}(\mathfrak{A})$ with barycentre $\omega \in \mathcal{S}(\mathfrak{A})$, i.e.

$$
\omega(A)=\int_{\mathcal{S}(A)} \varphi(A) d \nu(\varphi), \quad A \in \mathfrak{A} .
$$

For any selfadjoint elements $A, B \in \mathfrak{A}$ we have

$$
\begin{gathered}
\Delta_{\omega}(A) \geq \int_{\mathcal{S}(\mathfrak{A})} \Delta_{\varphi}(A) d \nu(\varphi) \\
\Delta_{\omega}(A) \cdot \Delta_{\omega}(B) \geq \int_{\mathcal{S}(\mathfrak{A})} \Delta_{\varphi}(A) \Delta_{\varphi}(B) d \nu(\varphi) .
\end{gathered}
$$

Proof of Theorem 3.1. Let the state $\omega$ be definite on each $Q_{\mu \nu}$; then $\omega$ is definite on $\left[q_{0}, \ldots, q_{3}\right]$ (cf. (3.4)) and $\Delta_{\omega}\left(e_{j}\right)=\Delta_{\omega}\left(m_{j}\right)=0, j=1,2,3$, so that (3.11), (3.12) follow from (3.13), (3.14) of Proposition 3.2. If $\omega$ is any state in the domain of the $\left[q_{\mu}, q_{\nu}\right]$ 's, it suffices, by Proposition 3.3 and Appendix A, to write $\omega$ as the barycentre of a (regular) probability measure carried by states definite on the $Q_{\mu \nu}$ 's.

Since the $Q_{\mu \nu}$ are central, $f\left(Q_{\mu \nu}\right), f \in \mathcal{C}_{0}(\mathbb{R})$, will lie in the centre of the $C^{*-}$ algebra $\mathfrak{A}$ generated by $f\left(Q_{\mu \nu}\right)$ and $g\left(q_{\mu}\right), \mu, \nu=0,1,2,3, f, g \in \mathcal{C}_{0}(\mathbb{R})$. For each state $\omega$ on $\mathfrak{A}$ in the domain of the $\left[q_{\mu}, q_{\nu}\right]$ 's, the central decomposition of $\omega[20,4.8]$ will provide a (regular) measure carried by factor states, hence definite on $f\left(Q_{\mu \nu}\right)$ and in the domain of $\left[q_{\mu}, q_{\nu}\right]$ by Appendix A.

Proof of Proposition 3.2. If $A, B, C$ are selfadjoint operators with $[A, B]^{-}=i C$, then for each state $\omega$ in the domain of $[A, B]$ we have

$$
\omega\left(C^{2}\right)=\omega(C)^{2}+\left(\Delta_{\omega}(C)\right)^{2} \leq\left(2 \Delta_{\omega}(A) \cdot \Delta_{\omega}(B)\right)^{2}+\left(\Delta_{\omega} C\right)^{2} .
$$

If $\omega$ is as in the statement of the proposition we have $\omega\left(\left[q_{0}, \ldots, q_{3}\right]\right)= \pm 2$ and by (3.7),

$$
\operatorname{Re} \omega(\vec{e} \cdot \vec{m})=\frac{1}{2} \omega(\vec{e} \cdot \vec{m}+\vec{m} \cdot \vec{e})= \pm 1,
$$

so that $|\omega(\vec{e} \cdot \vec{m})| \geq 1$. Since by the Schwarz inequality

$$
\begin{aligned}
|\omega(\vec{e} \cdot \vec{m})| & \leq \sum_{j} \omega\left(e_{j}^{2}\right)^{1 / 2} \omega\left(m_{j}^{2}\right)^{1 / 2} \\
& \leq\left(\sum_{j} \omega\left(e_{j}^{2}\right)\right)^{1 / 2}\left(\sum_{j} \omega\left(m_{j}^{2}\right)\right)^{1 / 2},
\end{aligned}
$$

we get

$$
\omega(\vec{e})^{2} \cdot \omega\left(\vec{m}^{2}\right) \geq 1
$$


Since by (3.6), (3.9) $\omega\left(\vec{e}^{2}\right)=\omega\left(\vec{m}^{2}\right)$ we have $\omega\left(\vec{e}^{2}\right) \geq 1, \omega\left(\vec{m}^{2}\right) \geq 1$. If we recall definitions (3.3), (3.5) and apply (3.18), we get (3.13) and (3.14) using ( $a+b+\cdots+$ $c)^{2} \geq a^{2}+b^{2}+\cdots+c^{2}$ for non-negative $a, b, \ldots, c$.

Proof of Proposition 3.3. With $X \in \mathfrak{A}, X=X^{*}$, we have

$$
\Delta_{\omega}(X)^{2}=\omega\left((X-\omega(X) I)^{2}\right)=\int \varphi\left((X-\omega(X) \cdot I)^{2}\right) d \nu(\varphi) .
$$

But for each $\psi \in \mathcal{S}(\mathfrak{A})$ and $\lambda \in \mathbb{R}$ we have

$$
\begin{aligned}
\psi\left((X-\lambda \cdot I)^{2}\right) & =\psi\left((X-\psi(X) \cdot I)^{2}\right)+(\lambda-\psi(X))^{2} \\
& \geq \psi\left((X-\psi(X) \cdot I)^{2}\right)
\end{aligned}
$$

so that we also have

$$
\begin{aligned}
\Delta_{\omega}(X)^{2} & \geq \int \varphi\left((X-\varphi(X) \cdot I)^{2}\right) d \nu(\varphi) \\
& =\int\left(\Delta_{\varphi} X\right)^{2} d \nu(\varphi)
\end{aligned}
$$

Since $\nu$ is a probability measure, the constant function equal to 1 is square summable with unit $L^{2}$-norm and by the Schwarz inequality

$$
\begin{aligned}
\int \Delta_{\varphi} X d \nu(\varphi) & \leq\left(\int\left(\Delta_{\varphi} X\right)^{2} d \nu(\varphi)\right)^{1 / 2} \leq \Delta_{\omega} X \\
\int \Delta_{\varphi} A \cdot \Delta_{\varphi} B d \nu(\varphi) & \leq\left(\int\left(\Delta_{\varphi} A\right)^{2} d \nu(\varphi)\right)^{1 / 2} \cdot\left(\int\left(\Delta_{\varphi} B\right)^{2} d \nu(\varphi)\right)^{1 / 2} \\
& \leq \Delta_{\omega} A \cdot \Delta_{\omega} B
\end{aligned}
$$

where we have used (3.19) repeatedly.

We now want to consider realizations of (3.9) and (3.10) through operators on Hilbert space. As we have already indicated, the $q_{\mu}$ will be supposed to be selfadjoint operators such that $-i\left[q_{\mu}, q_{\nu}\right]$ have self-adjoint closures $Q_{\mu \nu}$ commuting strongly with $q_{\lambda}$ for all $\mu, \nu, \lambda$. We wish, however, to impose a further condition namely that the commutation relations between the $q_{\mu}$ 's can be integrated in Weyl form:

$$
e^{i \alpha_{\mu} q^{\mu}} e^{i \beta_{\mu} q^{\nu}}=e^{-(i / 2) \alpha_{\mu} Q^{\mu \nu} \beta_{\nu}} e^{i(\alpha+\beta)_{\mu} q^{\mu}} ; \quad \alpha, \beta \in \mathbb{R}^{4} .
$$

Such realizations will be termed regular.

We shall see in the next section that there is a well defined $C^{*}$-algebra $\mathcal{E}$ whose non-degenerate representations are in one-to-one correspondence with the regular realizations. $\mathcal{E}$ describes our Quantum Spacetime in the sense that it may be thought of as the space of continuous functions on the Quantum Spacetime vanishing at infinity.

The situation is therefore analogous to quantum mechanics where we have a $C^{*}$-algebra whose representations are in one-to-one correspondence with regular realizations of the canonical commutation relations. Furthermore, just as in quantum mechanics, there are other realizations and thus the possibility of defining 
$C^{*}$-algebras describing other natural classes of representations and hence other Quantum Spacetimes. Taking quantum mechanics as our guide, these possibilities will be ignored here.

An important role will be played by the joint spectrum of the commuting selfadjoint operators $Q_{\mu \nu}$. By (3.9) it is included in the set $\Sigma$ of all antisymmetric real 2-tensors $\sigma$ such that

$$
\sigma_{\mu \nu} \sigma^{\mu \nu}=0 ; \quad \frac{1}{4} \sigma_{\mu \nu}(* \sigma)^{\mu \nu}= \pm 1,
$$

i.e., writing $\sigma=(\vec{e}, \vec{m}) ; e_{j}=\sigma_{0 j}, m_{j}=\sigma_{l k}$, where $(j, l, k)$ is a cyclic permutation of $(1,2,3)$, such that

$$
\vec{e}^{2}=\vec{m}^{2} ; \quad(\vec{e} \cdot \vec{m})^{2}=1 .
$$

We have $\Sigma=\Sigma_{+} \cup \Sigma_{-}$, where

$$
\Sigma_{ \pm}=\left\{\sigma=(\vec{e}, \vec{m}) / \vec{e}^{2}=\vec{m}^{2}, \quad \vec{e} \cdot \vec{m}= \pm 1\right\} .
$$

We can introduce the (Lorentz frame dependent) Euclidean norm on $\Sigma$ by

$$
\|\sigma\|^{2} \equiv \frac{1}{2} \sum_{\mu<\nu} \sigma_{\mu \nu}^{2}=\frac{1}{2}\left(\vec{e}^{2}+\vec{m}^{2}\right)=\vec{e}^{2}=\vec{m}^{2} .
$$

Then $\|\sigma\| \geq 1$ for each $\sigma \in \Sigma$ and the unit sphere $\Sigma^{(1)}$ of $\Sigma$ is the compact manifold

$$
\Sigma^{(1)}=\{\sigma \in \Sigma /\|\sigma\|=1\}=\{\sigma=(\vec{e}, \vec{m}) \in \Sigma / \vec{e}= \pm \vec{m}\}=\Sigma_{+}^{(1)} \cup \Sigma_{-}^{(1)},
$$

i.e. $\Sigma_{ \pm}^{(1)}$ is homeomorphic to $S^{2}$.

If the four selfadjoint operators $q_{0}, \ldots, q_{3}$ obey the Quantum Conditions (3.9), (3.10) and $\omega$ is a state in the domain of the $Q_{\mu \nu}$ 's then, by the Spectral Theorem, $\omega$ defines a regular probability measure $\mu_{\omega}$ on $\Sigma$ s.t.

$$
\omega(f(Q))=\int_{\Sigma} f(\sigma) d \mu_{\omega}(\sigma), \quad f \in \mathcal{C}_{0}(\Sigma)
$$

3.4 Proposition. Under the hypothesis of Theorem 1, we have

$$
\Sigma_{\mu}\left(\Delta_{\omega} q_{\mu}\right)^{2} \geq \sqrt{2} \int_{\Sigma}\left(\|\sigma\|^{2}+1\right)^{1 / 2} d \mu_{\omega}(\sigma) .
$$

Proof. By (3.19) it sufficies to prove the above inequality for a state $\omega$ which is pure on the centre. $\mu_{\omega}$ is then a Dirac measure at a point $\sigma \in \Sigma$ and $\left|\omega\left(Q_{\mu \nu}\right)\right|=\left|\sigma_{\mu \nu}\right|$. Let $q_{\mu}^{\prime}=\pi_{\omega}\left(q_{\mu}\right)-\omega\left(q_{\mu}\right) \cdot I$. For $\vec{a} \in \mathbb{R}^{3}$ we set $\vec{a} \cdot \vec{q}^{\prime}=\Sigma_{i=1}^{3} a_{i} q_{i}^{\prime}$ and find the commutation relations

$$
\left[q_{0}^{\prime}, \vec{a} \cdot \vec{q}^{\prime}\right]=i \vec{a} \cdot \vec{e} \cdot I, \quad\left[\vec{a} \cdot \vec{q}^{\prime}, \vec{b} \cdot \vec{q}^{\prime}\right]=i(\vec{a} \times \vec{b}) \cdot \vec{m} \cdot I .
$$

Now let $\{\vec{a}, \vec{b}, \vec{c}\}$ be an orthonormal basis of $\mathbb{R}^{3}$ with $\vec{a}=\vec{b} \times \vec{c}$. 
Then

$$
\begin{aligned}
\sum_{\mu}\left(\Delta_{\omega}\left(q_{\mu}\right)\right)^{2} & =\omega\left(q_{0}^{\prime 2}+\left(\vec{a} \cdot \vec{q}^{\prime}\right)^{2}+\left(\vec{b} \cdot \vec{q}^{\prime}\right)^{2}+\left(\vec{c} \cdot \vec{q}^{\prime}\right)^{2}\right) \\
& \geq 2 \Delta_{\omega}\left(q_{0}^{\prime}\right) \Delta_{\omega}\left(\vec{a} \cdot \vec{q}^{\prime}\right)+2 \Delta_{\omega}\left(\vec{b} \cdot \vec{q}^{\prime}\right) \Delta_{\omega}\left(\vec{c} \cdot \vec{q}^{\prime}\right) \\
& \geq\left|\omega\left(\left[q_{0}^{\prime}, \vec{a} \cdot \vec{q}^{\prime}\right]\right)\right|+\left|\omega\left(\left[\vec{b} \cdot \vec{q}^{\prime}, \vec{c} \cdot \vec{q}^{\prime}\right]\right)\right| \\
& =|\vec{a} \cdot \vec{e}|+|(\vec{b} \times \vec{c}) \cdot \vec{m}| \geq|\vec{a} \cdot(\vec{e} \pm \vec{m})|
\end{aligned}
$$

for $\sigma \in \Sigma_{+}$respectively.

The maximum over $\vec{a}$ is attained for $\vec{a}=\frac{\vec{e} \pm \vec{m}}{\|\vec{e} \pm \vec{m}\|}$, hence

$$
\Sigma_{\mu}\left(\Delta_{\omega}\left(q_{\mu}\right)\right)^{2} \geq\|\vec{e} \pm \vec{m}\|=\sqrt{2\left(\|\vec{e}\|^{2}+1\right)}=\sqrt{2\left(\|\sigma\|^{2}+1\right)}
$$

The last proposition sheds some light on the role of the manifold $\Sigma$. Very accurate measurements of the $q_{\mu}$ 's select states $\omega$ for which $\mu_{\omega}$ is essentially concentrated on $\Sigma^{(1)}$. In generic units, the unit sphere $\Sigma^{(1)}$ becomes the doubled sphere of radius $\lambda_{P}^{2}$

As we will discuss in the next section, if we consider generic states, the manifold $\Sigma$ survives in the classical limit $\lambda_{P} \rightarrow 0$. But if we limit ourselves to very well localized states, the effect of $\Sigma$ will be not directly visible in that limit. In the next section we will describe explicitly states with optimal localization where the quantity $\Sigma_{\mu}\left(\Delta_{\omega} q_{\mu}\right)^{2}$ actually reaches its minimal possible value.

We collect here some easy results on the manifold $\Sigma$ which are either obvious or proved in Appendix B.

I. $\Sigma$ is a homogeneous space of the full Lorentz group for the action

$$
\begin{gathered}
\Lambda \in L, \quad \sigma \in \Sigma \rightarrow \Lambda \sigma \Lambda^{T}=\sigma^{\prime} \in \Sigma, \\
\sigma_{\mu \nu}^{\prime}=\Lambda_{\mu}^{\mu^{\prime}} \Lambda_{\nu}^{\mu^{\prime}} \sigma_{\mu^{\prime} \nu^{\prime}} .
\end{gathered}
$$

Under the action $(3.24), \Sigma_{ \pm}$are $L_{+}^{\uparrow}$-homogeneous spaces; $\Sigma_{ \pm}$are connected. If $\vec{e}=\vec{m}$, the stabilizer of $\sigma=(\vec{e}, \vec{m})$ in $L_{+}^{\uparrow}$ consists precisely of boosts along $\vec{e}$ combined with rotations around $\vec{e}$. If $\vec{e}=\vec{m}$ is chosen as the third axis, the stabilizer of $\sigma$ is the image of the subgroup $D$ of diagonal matrices under the usual covering map $S L(2, \mathbb{C}) \rightarrow L_{+}^{\uparrow}$. Hence we have homeomorphisms and isomorphisms of $L_{+}^{\uparrow}$-homogeneous spaces:

$$
\Sigma_{+} \sim \Sigma_{-} \sim S L(2, \mathbb{C}) / D
$$

II. As a topological space, $\Sigma_{+}$is homeomorphic to $T S^{2}$, the tangent bundle of the unit sphere in $\mathbb{R}^{3}$. The two-sphere $S^{2}$, naturally embedded in $T S^{2}$, corresponds precisely to $\Sigma_{+}^{(1)}$, the Euclidean unit sphere of $\Sigma_{+}$. In particular, $S^{2}$ is a deformation retract of $\Sigma_{+}$.

III. There are Borel sections for the map of $L_{+}^{\uparrow}$ onto $\Sigma_{+}: \Lambda \in L_{+}^{\uparrow} \rightarrow \Lambda \sigma_{0} \Lambda^{T}, \sigma_{0}$ being a given point in $\Sigma_{+}$; we can choose such a section

$$
\sigma \in \Sigma_{+} \rightarrow \Lambda_{\sigma} \in L_{+}^{\uparrow}
$$




$$
\Lambda_{\sigma} \sigma_{0} \Lambda_{\sigma}^{T}=\sigma, \quad \sigma \in \Sigma_{+}
$$

to be continuous on the complement of a closed set $N$ with zero quasi-invariant measure. We can choose two such sections $\sigma \rightarrow \Lambda_{\sigma}, \sigma \rightarrow \Lambda_{\sigma}^{\prime}$, such that $N \cap N^{\prime}=\emptyset$, each of which can be written in the form

$$
\Lambda_{\sigma}=L_{\sigma} R_{\sigma}, \quad \sigma \in \Sigma_{+}
$$

where $L_{\sigma}$ is a boost, $R_{\sigma}$ a rotation and $\sigma \in \Sigma_{+} \rightarrow L_{\sigma} \in L_{+}^{\uparrow}$ is continuous.

Choosing $\sigma_{0} \in \Sigma_{+}$s.t. $\sigma_{0}=(\vec{e}, \vec{m}), \vec{e}=\vec{m}=(0,1,0)$, we have

$$
\left(\sigma_{0}^{\mu \nu}\right)=\left(\begin{array}{cc}
0 & -I \\
I & 0
\end{array}\right) \equiv s,
$$

where $I$ is the unit $2 \times 2$ matrix. Thus the symplectic form $\alpha, \beta \in \mathbb{R}^{4} \rightarrow \alpha_{\mu} \sigma_{0}^{\mu \nu} \beta_{\nu}$ can be written as $-\operatorname{Im}(\tilde{\alpha}, \tilde{\beta})$, where $\tilde{\alpha}=\left(\alpha_{0}+i \alpha_{2}, \alpha_{1}+i \alpha_{3}\right) \in \mathbb{C}^{2}$. In particular, $\sigma_{0}$ and hence any $\sigma \in \Sigma$, induces a non-degenerate symplectic form and is an invertible matrix.

A regular realization with $Q^{\mu \nu}=\sigma_{0}^{\mu \nu} \cdot I$ is given by the Schrödinger operators in the plane, $Q_{j}=$ multiplication by $s_{j}$ and $P_{j}=-i \frac{\partial}{\partial s_{j}}, j=1,2$, in $L^{2}\left(\mathbb{R}^{2}, d^{2} s\right)=H$, by setting

$$
q_{0}^{\sigma_{0}}=P_{1}, q_{1}^{\sigma_{0}}=P_{2} ; \quad q_{2}^{\sigma_{0}}=Q_{1} ; \quad q_{3}^{\sigma_{0}}=Q_{2}
$$

By von Neumann's uniqueness theorem, each regular irreducible realization is a (improper) Lorentz transform $q^{\sigma}$ of $q^{\sigma_{0}}, \sigma=\Lambda \sigma_{0} \Lambda^{T}=\sigma_{0 \Lambda}$, for some $\Lambda \in L$. By reduction theory every regular realization will be a direct integral of multiples of $q^{\sigma}$ 's.

A Poincaré-covariant realization can be easily constructed as follows. Denoting by $\bar{x} \in \bar{H}$ and $\bar{A}$ the complex conjugates of an element $x$ of a Hilbert space $H$ and of a linear operator $A$ on $H$, i.e. $\overline{A x}=\overline{A x}$, define

$$
\begin{gathered}
\tilde{q}^{\sigma}=q^{\sigma} \otimes I \text { on } H \otimes \bar{H} \\
P_{\mu}=-\left(\left(\sigma^{-1} q^{\sigma}\right)_{\mu} \otimes I+I \otimes\left(\sigma^{-1} \bar{q}^{\sigma}\right)_{\mu}\right) .
\end{gathered}
$$

It is easily checked that the $P_{\mu}$ are generators of a representation $\mathcal{U}_{\sigma}$ of $\mathbb{R}^{4}, \mathcal{U}_{\sigma}(a)=$ $e^{i P_{\mu} a^{\mu}}$, which is unitary, strongly continuous and induces translations on $\tilde{q}^{\sigma}$ :

$$
\mathcal{U}_{\sigma}(a)^{-1} \tilde{q}_{\mu}^{\sigma} \mathcal{U}_{\sigma}(a)=\tilde{q}_{\mu}^{\sigma}+a_{\mu} \cdot I
$$

We can now define, on the Hilbert space $\mathcal{H}=\int_{\oplus} H \otimes \bar{H} d \Lambda \cong H \otimes \bar{H} \otimes L^{2}(L)$, the operators

$$
\begin{gathered}
q_{\mu} \equiv \int_{\oplus}\left(\Lambda q^{\sigma}\right)_{\mu} d \Lambda ; \\
\mathcal{U}(a, I) \equiv \int_{\oplus} \mathcal{U}_{\sigma}\left(\Lambda^{-1} a\right) d \Lambda, \quad a \in \mathbb{R}^{4} ; \\
\mathcal{U}(0, \Lambda):(\mathcal{U}(0, \Lambda) x)\left(\Lambda^{\prime}\right)=x\left(\Lambda^{-1} \Lambda^{\prime}\right), \Lambda, \Lambda^{\prime} \in L, x \in \mathcal{H} ; \\
\mathcal{U}(a, \Lambda) \equiv \mathcal{U}(a, I) \mathcal{U}(0, \Lambda) .
\end{gathered}
$$


It is easily checked that (3.35) defines a unitary strongly continuous representation of the full Poincaré group and (3.32) a regular realization, where

$$
\mathcal{U}(a, \Lambda)^{-1} q_{\mu} \mathcal{U}(a, \Lambda)=(\Lambda q)_{\mu}+a_{\mu} \cdot I .
$$

By von Neumann uniqueness and uniqueness of the quasi-invariant regular measure class on $S L(2, \mathbb{C}) / D$, every regular realization, covariant in the sense of $(3.36)$, will have to be quasi-equivalent to (3.32).

We close this section with a remark on our Quantum Conditions (3.9), (3.10). We could as well have required that the two invariant combinations of the central operators $Q_{\mu \nu}$ appearing in (3.9) coincide with generic real multiples of the identity, say $Q_{\mu \nu} Q^{\mu \nu}=2 b \cdot I,\left(\frac{1}{4} Q_{\mu \nu}(* Q)^{\mu \nu}\right)^{2}=\left(a^{2}-b^{2}\right) \cdot I$, with $a \geq|b|$. The above discussion would have shown that for each state $\omega$, the number $\eta \equiv \omega\left(\vec{e}^{2}\right), \mu=$ $\omega\left(\vec{m}^{2}\right)$ fulfil $\mu \eta \geq a^{2}-b^{2}, \mu-\eta=2 b$. Hence $\eta \geq a-b, \mu \geq a+b$, leading as above to the uncertainty relations

$$
\begin{aligned}
& \Delta q_{0} \Sigma \Delta q_{j} \geq \frac{1}{2}(a-b), \\
& \Sigma \Delta q_{j} \Delta q_{k} \geq \frac{1}{2}(a+b) .
\end{aligned}
$$

Thus our choice $b=0, a=1$ is the obvious symmetric choice in natural units.

\section{Quantum Spacetime}

In this section we discuss the $C^{*}$-algebra describing Quantum Spacetime. This part of our discussion is not hard but still rather technical, and readers who are less mathematically minded might prefer to limit themselves to the statements of the theorems and then proceed to the discussion of localization and the classical limit following Theorem 4.2.

In view of the discussion towards the end of Sect. 3, we may expect that the $C^{*}$ algebra describing the Quantum Spacetime associated with the regular realizations of the Quantum Conditions (3.9), (3.10) (cf. Eq. (3.20)) is related to the normclosed algebra generated by

$$
\int f(\alpha) e^{i \alpha^{\mu} q_{\mu}} d^{4} \alpha ; \quad f \in L^{1}\left(\mathbb{R}^{4}, d^{4} \alpha\right)
$$

where $q_{\mu}$ is the realization (3.32).

In order to nail down the appropriate algebra, it is more instructive to follow von Neumann's approach to uniqueness and first introduce a Banach $*$-algebra $\mathcal{E}_{0}$ associated with the regular realizations.

Let us define $\mathcal{E}_{0}$ as the Banach space of continuous functions from $\Sigma$ to $L^{1}\left(\mathbb{R}^{4}, d^{4} \alpha\right)$ vanishing at infinity, equipped with the product, ${ }^{*}$, and norm:

$$
\begin{gathered}
(f \times g)(\sigma, \alpha)=\int f\left(\sigma, \alpha^{\prime}\right) g\left(\sigma, \alpha-\alpha^{\prime}\right) e^{(i / 2) \alpha_{\mu} \sigma^{\mu \nu} \alpha_{\nu}^{\prime}} d^{4} \alpha^{\prime}, \\
\left(f^{*}\right)(\sigma, \alpha)=\overline{f(\sigma,-\alpha)} \\
\|f\|_{0,1}=\sup _{\sigma}\|f(\sigma, \cdot)\|_{1}
\end{gathered}
$$


We define the action $\tau$ of $\mathcal{P}$ on $\mathcal{E}_{0}$ by

$$
\tau_{(a, \Lambda)}(f)(\sigma, \alpha)=f\left(\Lambda^{-1} \sigma \Lambda^{-1 T} ; \Lambda^{-1} \alpha\right) e^{-i a_{\mu} \alpha^{\mu}} \operatorname{det} \Lambda,
$$

where of course $\operatorname{det} \Lambda= \pm 1$.

The commutative $C^{*}$-algebra $\mathcal{C}(\Sigma)$ of bounded continuous functions on $\Sigma$ is embedded in the multiplier algebra of $\mathcal{E}_{0}$ by

$$
\begin{gathered}
g \in \mathcal{C}(\Sigma), f \in \mathcal{E}_{0} \rightarrow g f \in \mathcal{E}_{0}, \\
(g f)(\sigma, \alpha)=g(\sigma) f(\sigma, \alpha) .
\end{gathered}
$$

Every non-degenerate representation $\pi$ of $\mathcal{E}_{0}$ then determines a non-degenerate representation $\tilde{\pi}$ of $\mathcal{C}(\Sigma)$ s.t. (cf. (4.6))

$$
\tilde{\pi}(g) \pi(f)=\pi(g f)=\pi(f) \tilde{\pi}(g)
$$

and $\pi$ is irreducible only if $\exists \sigma \in \Sigma$ s.t.

$$
\tilde{\pi}(g)=g(\sigma) \cdot I
$$

By von Neumann uniqueness, the irreducible representations $\pi$ fulfilling (4.8) for a fixed $\sigma \in \Sigma$ are all equivalent to one another and to the representation of $\mathcal{E}_{0}$ determined by $q^{\sigma}$

$$
f \in \mathcal{E}_{0} \rightarrow \int d^{4} \alpha f(\sigma, \alpha) e^{i \alpha^{\mu} q_{\mu}^{\sigma}}
$$

where (cf. (3.29))

$$
q^{\sigma}=\Lambda q^{\sigma_{0}} \quad \text { if } \quad \sigma=\Lambda \sigma_{0} \Lambda^{T}, \quad \Lambda \in L .
$$

4.1 Theorem. There exists a unique $C^{*}$-norm on $\mathcal{E}_{0}$. The completion $\mathcal{E}$ is the $C^{*}$-algebra associated with a trivial continuous field of elementary algebras on $\Sigma$, i.e. it is isomorphic to $\mathcal{C}_{0}(\Sigma, \mathcal{K})$, where $\mathcal{K}$ is the $C^{*}$-algebra of all compact operators on a fixed separable infinite dimensional Hilbert space.

Proof. The representations (4.9), (4.10), for $\sigma \in \Sigma$, form a separating family, hence $\mathcal{E}_{0}$ admits a $C^{*}$-norm. Let \|\| denote the maximal $C^{*}$-norm and $\mathcal{E}$ the associated completion. A non-zero representation $\pi$ of $\mathcal{E}$ is irreducible iff $\pi \mid \mathcal{E}_{0}$ is irreducible, and hence iff $\pi \mid \mathcal{E}_{0}$ is unitarily equivalent to the representation given by (4.9), (4.10) for some $\sigma \in \Sigma$.

Therefore, for each $\sigma \in \Sigma$ there is an irreducible representation $\pi_{\sigma}$ of $\mathcal{E}$ satisfying (4.8) which is unique up to equivalence and we have

$$
\pi_{\sigma}(\mathcal{E})=\mathcal{K}\left(\mathcal{H}_{\pi_{\sigma}}\right), \quad \sigma \in \Sigma .
$$

If we embed $\mathcal{E}_{0}$ into the Banach $*-$ algebra $\tilde{\mathcal{E}}_{0}$ of bounded continuous functions from $\Sigma$ to $L^{1}\left(\mathbb{R}^{4}\right)$ with product, ${ }^{*}$, and norm given by (4.2), (4.3), (4.4), then clearly $\mathcal{E}_{0} \subset \tilde{\mathcal{E}}_{0} \subset M\left(\mathcal{E}_{0}\right)$, the multiplier algebra of $\mathcal{E}_{0}$. The maximal $C^{*}$-norm $\|\cdot\|$ on $\mathcal{E}_{0}$ 
induces a $C^{*}$-norm, still denoted by $\|\cdot\|$, on $\tilde{\mathcal{E}}_{0}$. If $\tilde{\mathcal{E}}$ is the completion of $\tilde{\mathcal{E}}_{0}$ in that norm, we have

$$
\mathcal{E} \subset \tilde{\mathcal{E}} \subset M(\mathcal{E})
$$

and, for each $\sigma \in \Sigma, \pi_{\sigma}(\mathcal{E})=\pi_{\sigma}(\tilde{\mathcal{E}})=\mathcal{K}\left(\mathcal{H}_{\pi_{\sigma}}\right)$.

We will show that the bundle $\left\{\pi_{\sigma}(\tilde{\mathcal{E}}) ; \sigma \in \Sigma\right\}$ equipped with the continuous fields $\left\{\left\{\pi_{\sigma}(A): \sigma \in \Sigma\right\}, A \in \tilde{\mathcal{E}}\right\}$ is a trivial continuous field of elementary algebras. $\mathcal{E}$ is then the associated $C^{*}$-algebra, i.e. the $C^{*}$-algebra of continuous fields vanishing at infinity, and is isomorphic to $\mathcal{C}_{0}(\Sigma, \mathcal{K})$.

To this end it suffices to show that our bundle is locally trivial and that there is a continuous field of one-dimensional projections (cf. [21, ch. 10, 7.6, 7.15, 8.4]).

We therefore choose the representations $\pi_{\sigma}, \sigma \in \Sigma$ in an appropriate way. For each $\sigma \in \Sigma$, let $f_{0}(\sigma, \cdot) \in L^{1}$ be the von Neumann element in the fibre of $\mathcal{E}_{0}$ at $\sigma$ corresponding to the selfadjoint projection on the ground state of the harmonic oscillator. Namely, if $\sigma \in \Sigma \rightarrow \Lambda_{\sigma} \in L$ is a section as in Sect. 3, III (cf. eq. (3.29) and II, Appendix B), we define $f_{0}$ by

$$
\begin{gathered}
f_{0}(\sigma, \alpha)=\frac{1}{(2 \pi)^{4}} e^{-\frac{1}{4}(\alpha, \alpha)_{\sigma}}, \quad \sigma \in \Sigma, \quad \alpha \in \mathbb{R}^{4}, \\
(\alpha, \alpha)_{\sigma}=\left(\Lambda_{\sigma}^{-1} \alpha, \Lambda_{\sigma}^{-1} \alpha\right)=\left(L_{\sigma}^{-1} \alpha, L_{\sigma}^{-1} \alpha\right), \quad \sigma \in \Sigma, \alpha \in \mathbb{R}^{4},
\end{gathered}
$$

where $(\alpha, \alpha)=\sum_{i=0}^{3} \alpha_{i}^{2}$ and we used the specific form of our section $\Lambda_{\sigma}=L_{\sigma} R_{\sigma}$, where $R_{\sigma}$ is a rotation thus leaving the Euclidean scalar product invariant.

Since the Lorentz group acts continuously on $L^{1}\left(\mathbb{R}^{4}\right): f \in L^{1}\left(\mathbb{R}^{4}\right) \rightarrow f_{L}$ : $f_{L}(\alpha)=f\left(L^{-1} \alpha\right)$, and $\sigma \in \Sigma \rightarrow L_{\sigma}$ is continuous (cf. Appendix B), $\sigma \rightarrow f_{0}(\sigma, \cdot) \in$ $L^{1}$ is continuous, where $f_{0}(\sigma, \cdot)=f_{0}\left(\sigma_{0}, \cdot\right)_{L_{\sigma}}$.

Now $\pi_{\sigma}\left(f_{0}\right)$ is a selfadjoint projection of rank 1 [22], so that $\left\{\pi_{\sigma}\left(f_{0}\right) ; \sigma \in \Sigma\right\}$ is a continuous field of rank one projections.

Furthermore the following relation holds for each $\sigma \in \Sigma$ :

$$
\left(f_{0} \times f \times f_{0}\right)(\sigma, \cdot)=\omega_{\sigma}(f) f_{0}(\sigma, \cdot), f \in \tilde{\mathcal{E}}_{0},
$$

and defines a state $\omega_{\sigma}$ on $\tilde{\mathcal{E}}$. From now on let $\left(\pi_{\sigma}, \mathcal{H}_{\sigma}, \xi_{\sigma}\right)$ be the GNS construction for $\omega_{\sigma}$. We have only to show that our continuous field is locally trivial.

Let $\Lambda_{1}, \Lambda_{2}$ be two sections as in III, Sect. 3, continuous on $\Sigma_{1}, \Sigma_{2}$ resp. where $\Sigma_{1}, \Sigma_{2}$ is an open covering of $\Sigma$ (cf. Appendix B). Define an isomorphism $\rho_{i}$ of $\mathcal{C}_{0}\left(\Sigma_{i}, L^{1}\right)$ regarded as the tensor product of $\mathcal{C}_{0}\left(\Sigma_{i}\right)$ and the fibre of $\mathcal{E}_{0}$ at $\sigma_{0}$ (cf. (4.2)) onto $\mathcal{C}_{0}\left(\Sigma_{i}, L^{1}\right)$ regarded as a subalgebra of $\mathcal{E}$ by

$$
\rho_{i}(f)(\sigma, \alpha)=f\left(\sigma, \Lambda_{i \sigma}^{-1} \alpha\right) ;
$$

one checks that $\rho_{i}$ is a $*$-isomorphism defining a trivialization of our field on $\Sigma_{i}$.

Remark. The triviality of our continuous field would follow directly if we could define a map, continuous in the appropriate sense, assigning to each $\sigma \in \Sigma$ a regular solution $\tilde{q}^{\sigma}$ s.t. $\left[\tilde{q}_{\mu}^{\sigma}, \tilde{q}_{\nu}^{\sigma}\right]=i \sigma_{\mu \nu} \cdot I$. We might tentatively define $\tilde{q}^{\sigma}$ as linear combinations of the fixed choice $q^{\sigma_{0}}$ of Eq. (3.29) but this approach fails, because the bundle $\Lambda \in L \rightarrow \sigma_{\Lambda} \in \Sigma$ is non-trivial and the symplectic group is not simply 
connected. However, we have the further possibility of deforming $q^{\sigma_{0}}$ to $U q^{\sigma_{0}} U^{-1}$, where $U$ is an element of the unitary group of $H$ using the fact that this group is contractible (cf. [21, Chap. 10]).

It follows from the proof of Theorem 4.1 that each $C^{*}$-seminorm on $\mathcal{E}$ has the form

$$
\|f\|_{S}=\sup _{\sigma \in S}\left\|\pi_{\sigma}(f)\right\|,
$$

for some closed subset $S$ of $\Sigma$. Since $\Sigma$ is a homogeneous space, the unique $C^{*}$-norm is also the only non-zero $\tau$-invariant $C^{*}$-seminorm, and $\tau$ extends to a strongly continuous action, still denoted by $\tau$, of the full Poincaré group by automorphisms of $\mathcal{E}$.

The quasiequivalence classes of representations of $\mathcal{E}$ are labelled by the regular measure classes on $\Sigma$. A covariant representation $\pi$ of $\mathcal{E}$ obviously yields a covariant representation $\tilde{\pi}$ of $\mathcal{C}_{0}(\Sigma)$ and is hence associated with a quasi-invariant regular measure on $\Sigma$. Since there is only one invariant measure class, it follows that there is only one quasiequivalence class of representations $\pi$ of $\mathcal{E}$ s.t. $(\pi, \mathcal{U})$ is a covariant representation of $(\mathcal{E}, \tau)$ for some representation $\mathcal{U}$ of the full Poincaré group.

The $C^{*}$-algebra $\mathcal{E}$ of Theorem 4.1 does indeed describe the Quantum Spacetime associated with the class of regular solutions of the Quantum Conditions (3.9), (3.10), in the sense of the previous section; namely:

4.2 Theorem. For each $f_{1} \in \mathcal{C}_{0}(\Sigma)$ and $f_{2} \in L^{1}\left(\mathbb{R}^{4}\right)$ let $f_{1} \otimes f_{2} \in \mathcal{E}_{0} \subset \mathcal{E}$ be the function $\sigma \in \Sigma \rightarrow f_{1}(\sigma) f_{2} \in L^{1}\left(\mathbb{R}^{4}\right)$. We can define selfadjoint operators $q_{\mu}$, $Q_{\mu \nu}$, affiliated to $\mathcal{E}$ with support of definition I (Appendix A), by setting, for each nondegenerate representation $\pi$ of $\mathcal{E}$,

$$
\begin{gathered}
\pi\left(f_{1} \otimes f_{2}\right)=f_{1}(\pi(Q)) \int f_{2}(\alpha) e^{i \alpha_{\mu} \pi\left(q^{\mu}\right)} d^{4} \alpha \\
f_{1} \in \mathcal{C}_{0}(\Sigma), f_{2} \in L^{1}\left(\mathbb{R}^{4}\right) .
\end{gathered}
$$

These operators obey the Quantum Conditions since

$$
\left[q_{\mu}, q_{\nu}\right]^{-}=i Q_{\mu \nu}
$$

the $Q_{\mu \nu}$ commute and joint spectrum of $\left\{Q_{\mu \nu}, \mu, \nu=0, \ldots, 3\right\}=\Sigma$ (cf. IV, Appendix $A$ ).

Moreover, for each element $(a, \Lambda)$ of the full Poincaré group, we have

$$
\tau_{(a, \Lambda)}^{-1}\left(q_{\mu}\right)=(\Lambda q+a \cdot I)_{\mu}
$$

where the automorphisms of $\mathcal{E}$ act on selfadjoint operators affiliated to $\mathcal{E}$ as described in Appendix A III.

We will refrain from spelling out the easy proof of this theorem and limit ourselves to the remark that, by the relations defining $q_{\mu}$ and $Q_{\mu \nu}$, if two representations $\pi_{1}$, $\pi_{2}$ of $\mathcal{E}$ fulfill $\pi_{1}\left(q_{\mu}\right)=\pi_{2}\left(q_{\mu}\right)$, then $\pi_{1}\left(Q_{\mu \nu}\right)=\pi_{2}\left(Q_{\mu \nu}\right)$, and $\pi_{1}, \pi_{2}$ agree on a total subset of $\mathcal{E}_{0}$, hence $\pi_{1}=\pi_{2}$ on $\mathcal{E}$. 
In view of Theorem 4.1 we can rewrite Eq. (4.17) as an identity in $\mathcal{E}$ involving the selfadjoint operators $q_{\mu}, Q_{\mu \nu}$ affiliated to $\mathcal{E}$ :

$$
f_{1} \times f_{2}=f_{1}(Q) \int f_{2}(\alpha) e^{i \alpha_{\mu} q^{\mu}} d^{4} \alpha .
$$

We next discuss briefly the physical meaning of the state space $\mathcal{S}(\mathcal{E})$. We interpret each state $\omega \in \mathcal{S}(\mathcal{E})$ as specifying the localization of events. Positivity of $\omega$ and the commutation relations prevent absolute precision in the localization, but there are states $\omega$ having optimal localization properties compatible with Proposition 3.4. The associated measure $\mu_{\omega}$ on $\Sigma$ will be concentrated on the unit sphere $\Sigma^{(1)}$, i.e. on the base $S^{2} \times\{ \pm 1\}$ of $T S^{2} \times\{ \pm 1\} \sim \Sigma$. The associated operators $q_{\mu}^{\sigma}, \sigma \in \Sigma^{(1)}$, will be obtained from the solution (3.29), associated to $\sigma_{0}: \sigma_{0}^{\mu \nu}=\left(\begin{array}{cc}0 & -I \\ I & 0\end{array}\right)$, by an (improper) rotation.

As is well known from elementary Quantum Mechanics, the minimum of the quantity (cf. (3.29))

$$
\Sigma_{\mu}\left(\Delta q_{\mu}^{\sigma}\right)^{2}=\left(\Delta Q_{1}\right)^{2}+\left(\Delta P_{1}\right)^{2}+\left(\Delta Q_{2}\right)^{2}+\left(\Delta P_{2}\right)^{2}
$$

is actually 2, and is attained on states which are translates of the ground state of the Hamiltonian $H$ of the harmonic oscillator in 2 dimensions,

$$
H=\frac{1}{2}\left(P_{1}^{2}+Q_{1}^{2}+P_{2}^{2}+Q_{2}^{2}\right)=\frac{1}{2} \Sigma_{\mu}\left(q_{\mu}^{\sigma}\right)^{2} .
$$

Therefore, we can parametrize the states on $\mathcal{E}$ with optimal localization by a vector $x$ in Minkowski space and a measure $\mu$ on $\Sigma$ carried by $\Sigma^{(1)}$ and define the associated state $\omega$ by its restriction to $\mathcal{E}_{0}$ :

$$
\begin{gathered}
\omega(f)=\int d^{4} \alpha d \mu(\sigma) f(\sigma, \alpha) e^{i \alpha_{\mu} x^{\mu}-\frac{1}{2} \Sigma_{\mu} \alpha_{\mu}^{2}}, \\
f \in \mathcal{C}_{0}\left(\Sigma, L^{1}\left(\mathbb{R}^{4}\right)\right)=\mathcal{E}_{0},
\end{gathered}
$$

so that $\omega$ is specified by the values of its normal extension $\tilde{\omega}$ to the multiplier algbra through

$$
\tilde{\omega}\left(f(Q) e^{i \alpha_{\mu} q^{\mu}}\right)=e^{i \alpha_{\mu} x^{\mu}-\frac{1}{2} \Sigma_{\mu} \alpha_{\mu}^{2}} \int f(\sigma) d \mu(\sigma), \quad \alpha \in \mathbb{R}^{4}, f \in \mathcal{C}(\Sigma) .
$$

We close this section with a remark on the classical limit $\lambda_{P} \rightarrow 0$.

In generic units, the twisting factor in the product (4.2) of $\mathcal{E}_{0}$ takes the form

$$
e^{\frac{i}{2} \lambda_{P}^{2} \alpha_{\mu} \sigma^{\mu \nu} \alpha_{\nu}^{\prime}}
$$

so that, as $\lambda_{P} \rightarrow 0$, our algebra $\mathcal{E}$ deforms to

$$
\mathcal{C}_{0}\left(\mathbb{R}^{4}\right) \otimes \mathcal{C}_{0}(\Sigma)=\mathcal{C}_{0}\left(\mathbb{R}^{4}\right) \otimes \mathcal{C}(\{ \pm 1\}) \otimes \mathcal{C}_{0}\left(\Sigma_{+}\right),
$$

that is our non-commutative space deforms to the (commutative) space

$$
\mathbb{R}^{4} \times\{ \pm 1\} \times \Sigma_{+} .
$$


The factor (4.22) corresponds to writing the Quantum Conditions on $q_{\mu}$, in generic units, in the form

$$
\begin{gathered}
{\left[q_{\mu}, q_{\nu}\right]=i \lambda_{P}^{2} \tilde{Q}_{\mu \nu},} \\
\tilde{Q}_{\mu \nu}(\sigma)=\sigma_{\mu \nu}, \quad \sigma \in \Sigma,
\end{gathered}
$$

where the $\tilde{Q}_{\mu \nu}$ defined this way are selfadjoint operators affiliated to $\mathcal{C}_{0}(\Sigma)$.

Equation (4.23) implies that the large dilation limit and the classical limit coincide. If we were prepared to violate this condition we could write

$$
\frac{1}{4}\left[q_{0}, \ldots, q_{3}\right]^{2}=\lambda_{P}^{8} \cdot I, \quad Q_{\mu \nu} Q^{\mu \nu}=0
$$

in place of (4.23) and the $\lambda_{P} \rightarrow 0$ limit would give a dilation covariant Quantum Spacetime, defined by

$$
\begin{gathered}
{\left[q_{\mu}, q_{\nu}\right]=i \mathcal{R}_{\mu \nu},} \\
\mathcal{R}_{\mu \nu}(\sigma)=\sigma_{\mu \nu}, \quad \sigma \in \Sigma_{0} .
\end{gathered}
$$

$\Sigma_{0}$ is the set of all real antisymmetric 2 -tensors such that

$$
\sigma_{\mu \nu} \sigma^{\mu \nu}=\sigma_{\mu \nu}(* \sigma)^{\mu \nu}=0
$$

Now $\Sigma_{0}$ is connected and is a single orbit under $L_{+}^{\uparrow}$ of any $\sigma \in \Sigma_{0}$; for each $\sigma \in \Sigma_{0}$, $\sigma=(\vec{e}, \vec{m})$ with $\vec{e}^{2}=\vec{m}^{2}, \vec{e} \cdot \vec{m}=0$. For each $\lambda>0$, an appropriate boost along $\vec{e} \times \vec{m}$ will change $\sigma=(\vec{e}, \vec{m})$ to $\lambda \sigma$. The symplectic form defined by $\sigma \in \Sigma_{0}$ is now degenerate, $\sigma \cong\left(\begin{array}{cc}0 & -1 \\ 1 & 0\end{array}\right) \oplus\left(\begin{array}{ll}0 & 0 \\ 0 & 0\end{array}\right)$. 


\section{Calculus on the Quantum Spacetime}

Let $\tilde{\mathcal{E}}$ denote, as before, the $C^{*}$-algebra of continuous bounded functions from $\Sigma$ to $\mathcal{K}$, and $Z \subset M(\tilde{\mathcal{E}})$ the Abelian $C^{*}$-algebra of bounded complex continuous functions on $\Sigma$.

With $f \in L^{1}\left(\mathbb{R}^{4}\right)$ let $\hat{f}=\mathcal{F} f \equiv g$ and $f=\overline{\mathcal{F}} g=\check{g}$ denote the Fourier transform and its inverse.

For each $f \in \mathcal{F} L^{1}\left(\mathbb{R}^{4}\right)$ we can define the function $f(q)$ of the quantum coordinates $q_{\mu}$ as an element of $\tilde{\mathcal{E}}$ by

$$
f(q) \equiv \int \check{f}(\alpha) e^{i q_{\mu} \alpha^{\mu}} d^{4} \alpha, \quad f \in \mathcal{F} L^{1}\left(\mathbb{R}^{4}\right) .
$$

Spacetime translations act as automorphisms $\tau_{a}, a \in \mathbb{R}^{4}$, such that (cf. Sect. 3,4)

$$
\tau_{a}(f(q))=f(q-a \cdot I), \quad a \in \mathbb{R}^{4} .
$$

We can now define spacetime derivatives as in Minkowski space as minus the infinitesimal generator of translations, i.e.

$$
\left.\partial_{\mu} f(q) \equiv \frac{\partial}{\partial a_{\mu}} f(q+a \cdot I)\right|_{a=0} .
$$

If we take the product of $n$ operators $f_{1}(q), \ldots, f_{n}(q)$ defined as in (5.1), we get another function of $q$ which on the one side is distinct from the pointwise product $f_{1} \ldots f_{n}$ evaluated on $q$ and on the other is no longer $\mathbb{C}$-valued but $Z$-valued in general. Both facts are apparent from the explicit formula which is an immediate consequence of (5.1):

$$
f_{1}(q) \ldots f_{n}(q)=\left(\check{f}_{1} \times \cdots \times \check{f}_{n}\right)^{\wedge}(q),
$$

where the twisted convolution $\times$ depends on $Q_{\mu \nu}$ :

$$
\begin{gathered}
\left(h \times h^{\prime}\right)(\alpha)=\int h\left(\alpha^{\prime}\right) h^{\prime}\left(\alpha-\alpha^{\prime}\right) e^{i / 2 \alpha Q \alpha^{\prime}} d^{4} \alpha, \\
\alpha Q \alpha^{\prime} \equiv \alpha_{\mu} Q^{\mu \nu} \alpha_{\nu}^{\prime} ; \quad h, h^{\prime} \in L^{1}\left(\mathbb{R}^{4}\right),
\end{gathered}
$$

so that

$$
\begin{aligned}
\left(\check{f}_{1} \times \cdots \times \check{f}_{n}\right)(k)=\int & d^{4} k_{1} \ldots d^{4} k_{n} \delta^{(4)}\left(k-\Sigma k_{j}\right) \cdot e^{i / 2 \Sigma_{j<l} k_{j} Q k_{l}} \\
& \times \check{f}\left(k_{1}\right) \ldots \check{f}\left(k_{n}\right) .
\end{aligned}
$$

In order to develop Quantum Field Theory on the Quantum Spacetime and to apply the conventional perturbation methods, it will be important to define the quantum analogues of the positive linear functional on $L^{1}\left(\mathbb{R}^{4}\right) \cap \mathcal{F} L^{1}\left(\mathbb{R}^{4}\right)$ given by the total integral and the integral over space at a fixed time $t$ :

$$
f \in L^{1} \cap \mathcal{F} L^{1} \rightarrow \int f(x) d^{4} x=\check{f}(0),
$$




$$
f \in L^{1} \cap \mathcal{F} L^{1} \rightarrow \int_{x_{0}=t} f(t, \vec{x}) d^{3} x=\int e^{i t k_{0}} \check{f}\left(k_{0}, \overrightarrow{0}\right) d k_{0} .
$$

Define the $Z$-valued trace $\operatorname{Tr}$ on $\tilde{\mathcal{E}}$ by

$$
(\operatorname{Tr}(X))(\sigma)=\operatorname{tr} X(\sigma), \quad \sigma \in \Sigma,
$$

where $\operatorname{tr}$ is the usual trace on $L^{1}(\mathcal{B}(\mathcal{H})) \subset \mathcal{K}(\mathcal{H})$.

If $f \in L^{1}\left(\mathbb{R}^{4}, Z\right) \cap \mathcal{F} L^{1}\left(\mathbb{R}^{4}, Z\right)$ the formal analogue of (5.7), (5.8) can be defined by

$$
\begin{aligned}
\int d^{4} q f(q) \equiv \int f(x) d^{4} x=\check{f}(0)=\operatorname{Tr} f(q), \\
\begin{aligned}
\int_{q_{0}=t} f(q) d^{3} q & \equiv \int e^{i k_{0} t} \check{f}\left(k_{0}, \overrightarrow{0}\right) d k_{0} \\
& =\lim _{m} \operatorname{Tr}\left(f_{m}(q)^{*} f(q) f_{m}(q)\right) .
\end{aligned}
\end{aligned}
$$

Note that (5.10), (5.11) are consistent since

$$
\int d t \int_{q_{0}=t} f(q) d^{3} q=\int f(q) d^{4} q .
$$

Positivity can be established either directly, using the first line of (5.11) as a definition and (5.5) to show that

$$
\int_{q_{0}=t} f(q)^{*} f(q) d^{3} q \geq 0
$$

where of course $f(q)^{*}=\bar{f}(q)$ as a consequence of the definition (5.1); or else by showing that the second equality in (5.11) does hold for an appropriate sequence $f_{m}$. To this end it suffices to choose real functions $f_{m}$ such that $f_{m}^{2}$ approximates the constant function one on space times the Dirac measure at time $t$. Then, using (5.6) for $n=3$, we get

$$
\begin{aligned}
\operatorname{Tr}_{m}(q)^{*} f(q) f_{m}(q) & =\operatorname{Tr} f_{m}(q) \bar{f}_{m}(q) f(q)=\left(\check{f}_{m} \times \check{f}_{m} \times f\right)(0) \\
& =\int d^{4} k_{1} d^{4} k_{2} d^{4} k_{3} \delta^{(4)}\left(\Sigma k_{j}\right) \cdot e^{(i / 2) \Sigma_{j<l} k_{j} Q k_{l}} \check{f}_{m}\left(k_{1}\right) \check{f}_{m}\left(k_{2}\right) \check{f}\left(k_{3}\right) \\
& =\int d^{4} k_{1} d^{4} k_{2} e^{(i / 2) k_{1} Q k_{2}} \check{f}_{m}\left(k_{1}\right) \check{f}_{m}\left(k_{2}\right) \check{f}\left(-\left(k_{1}+k_{2}\right)\right)
\end{aligned}
$$

where $\check{f}_{m} * \check{f}_{m}$ approximates $\delta^{(3)}(\vec{k}) \cdot e^{-i k_{0} t}$, so that $e^{(i / 2) k_{1} Q k_{2}}$ can be replaced by $I$ in the limit, since $Q_{00}=0$, and the expression (5.12) approximates $\int d k_{0} \check{f}\left(k_{0}, \overrightarrow{0}\right)$ $e^{-i k_{0} t}$, as desired. The positivity of the weight (5.11) can be interpreted in the light of our spacetime uncertainties which must hold for any positive functional and are compatible with absolute precision in the measurement of time together with complete lack of knowledge of the space coordinates. On the contrary, the functional $f(q) \rightarrow f(x), x \in \mathbb{R}^{4}$, for instance, is not positive on $\tilde{\mathcal{E}}$.

In order to obtain a $\mathbb{C}$-valued functional we must integrate over $Z$ too. If the result is to agree with the classical definition for functions taking values in $\mathbb{C}$. 
$I \subset Z$, we must use a normalized state on $Z$. Unfortunately there is no obvious Lorentz invariant choice since $L_{+}^{\uparrow}$ is not amenable. Motivated by our discussion of localization, we will choose the state defined by integration over the unit sphere $\Sigma^{(1)}$ with the normalized Lebesgue measure $d \sigma$. Of course the result will be rotation but not Lorentz invariant.

We first analyze the $Z$-valued weight $\int_{q_{0}=t} d^{3} q$ in more detail. In fact, in order be able to define an interaction Hamiltonian we will need expressions like

$$
\int_{q_{0}=t} f_{1}(q) \ldots f_{n}(q) d^{3} q
$$

Combining (5.6) with (5.11) we see that (5.13) is given by

$$
\int \mathcal{D}_{n}\left(x_{1}, \ldots, x_{n} ; t\right) f_{1}\left(x_{1}\right) \ldots f_{n}\left(x_{n}\right) d^{4} x_{1} \ldots d^{4} x_{n}
$$

where the $Z$-valued kernel $\mathcal{D}_{n}$ is given by

$$
\begin{aligned}
\mathcal{D}_{n}\left(x_{1}, \ldots, x_{n} ; t\right)=\int & d k_{0} d^{4} k_{1} \ldots d^{4} k_{n} e^{-i \Sigma_{j} k_{j} x_{j}} e^{i k_{0} t} \delta^{(1)}\left(k_{0}-\Sigma k_{i 0}\right) . \\
& \delta^{(3)}\left(\Sigma \vec{k}_{i}\right) e^{(i / 2) \Sigma_{j<l} k_{j} Q_{k_{l}}} .
\end{aligned}
$$

The explicit expressions are (cf. Appendix C):

$$
\begin{gathered}
\mathcal{D}_{2 n+1}\left(x_{1}, \ldots, x_{2 n+1} ; t\right)=\pi^{-4 n} e^{2 i \Sigma_{j \leq l \leq n}\left(x_{2 j}-x_{2 j-l}\right) Q^{-1}\left(x_{2 l+1}-x_{2 l}\right)} . \\
\delta\left(\sum_{j=1}^{2 n+1}(-1)^{j}\left(x_{j}^{0}-t\right)\right)
\end{gathered}
$$

and

$$
\begin{gathered}
\mathcal{D}_{2 n+2}\left(x_{1}, \ldots, x_{2 n+2} ; t\right)=\pi^{-4 n} \frac{1}{2 \pi} \int d \eta e^{i \eta \Sigma_{j=1}^{2 n+1}(-1)^{j}\left(x_{j}^{0}-t\right)} . \\
e^{2 i \Sigma_{j \leq l \leq n}\left(x_{2 j}-x_{2 j-1}\right) Q^{-1}\left(x_{2 l+1}-x_{2 l}\right)} \delta^{(4)}\left(\sum_{j=1}^{n+1}\left(x_{2 j}-x_{2 j-1}\right)-\frac{1}{2} Q(\eta, \overrightarrow{0})\right) .
\end{gathered}
$$

The kernel $\mathcal{D}_{n}$ describes the non-locality introduced by the quantum nature of spacetime as a deviation of (5.13) from the classical expression $\int f_{1}(\vec{x}, t) \ldots f_{n}(\vec{x}, t) d^{3} x$. We limit ourselves to the explicit expressions for $n=2,3$, which will be of particular importance in the next section. For $n=2$ we get

$$
\int_{q_{0}=t} f_{1}(q) f_{2}(q) d^{3} q=(2 \pi)^{-1} \int f_{1}(x) f_{2}(x+Q(\eta, \overrightarrow{0})) e^{-i \eta\left(t-x_{0}\right)} d^{4} x d \eta .
$$

Note that integrating over $t$ gives

$$
\int d t \int_{q_{0}=t} f_{1}(q) f_{2}(q) d^{3} q \equiv \int f_{1}(q) f_{2}(q) d^{3} q=\int f_{1}(x) f_{2}(x) d^{4} x
$$


so that there is no nonlocal effect in the spacetime integral when $n=2$.

The nonlocal effects also disappear from the space integral at fixed time if we restrict $f_{1}, f_{2}$ to be solutions of the Klein-Gordon equation $\left(\square+m^{2}\right) f=0$ and evaluate the usual scalar product

$$
\begin{aligned}
(f, g) & \equiv \int_{x_{0}=t} \bar{f}(x) \stackrel{\leftrightarrow}{\partial_{0}} g(x) d^{3} x \\
& =\int_{x_{0}=t}\left(\frac{\overline{\partial f}}{\partial x_{0}}(x) g(x)-\overline{f(x)} \frac{\partial g}{\partial x_{0}}(x)\right) d^{3} x
\end{aligned}
$$

Indeed we have for any such $f$ and $g$ and for each $t$,

$$
\int_{q_{0}=t} \overline{f(q)} \overleftrightarrow{\partial_{0}} g(q) d^{3} q=(f, g) \cdot I
$$

To prove (5.17) it suffices to note that, by (5.16), the l.h.s. can be written as

$$
(2 \pi)^{-1 / 2} \int d x_{0} d \eta e^{-i \eta\left(t-x_{0}\right)} \int d^{3} x f(x) \overleftrightarrow{\partial_{0}} g_{\eta}(x)
$$

where $g_{n}(x) \equiv g(x+Q(\eta, \overrightarrow{0}))$ is again a solution of the Klein-Gordon equation. Therefore the $\int d^{3} x$ does not depend on $x_{0}$ and integrating over $x_{0}$ gives the Dirac measure in the variable $\eta$. Integrating over $\eta$ replaces $g_{\eta}$ by $g_{0}=g$ and $(5.17)$ equals $(f, g) \cdot I$ as desired.

The calculation for $n=3$ gives

$$
\mathcal{D}_{3}\left(x_{1}, x_{2}, x_{3} ; t\right)=\left(\frac{1}{\pi}\right)^{4} \delta\left(t-x_{30}-\left(x_{10}-x_{20}\right)\right) e^{2 i\left(x_{2}-x_{1}\right) Q^{-1}\left(x_{3}-x_{2}\right)}
$$

so that

$$
\begin{gathered}
\int_{q_{0}=t} f_{1}(q) f_{2}(q) f_{3}(q) d^{3} q=\left(\frac{1}{\pi}\right)^{4} \int d^{4} a d^{4} b \int_{x_{0}=t+(b-a)_{0}} f_{1}(x+a) f_{2}(x+b) f_{3}(x) \\
\times e^{2 i a Q^{-1} b} d^{3} x
\end{gathered}
$$

Thus the non-local effects are now visible in the full spacetime integral, too, as we see by integrating (5.20) over $t$ :

$$
\begin{gathered}
\int f_{1}(q) f_{2}(q) f_{3}(q) d^{4} q= \\
\left(\frac{1}{\pi}\right)^{4} \int d^{4} a d^{4} b d^{4} x f_{1}(x+a) f_{2}(x+b) f_{3}(x) e^{2 i a Q^{-1} b} .
\end{gathered}
$$

It is instructive to write (5.20) in generic units. To this end we must replace $a_{\mu}$ by $\lambda_{P} a_{\mu}, b_{\mu}$ by $\lambda_{P} b_{\mu}$ and $Q^{-1}$ by $\lambda_{P}^{-2} K$, where $a_{\mu}, b_{\mu}$ and $K: \sigma \in \Sigma \rightarrow K(\sigma)=$ $\left(\left(\sigma^{\mu \nu}\right)_{\mu, \nu=0, \ldots, 3}\right)^{-1}$ are dimensionless.

We get

$$
\int_{q_{0}=t} f_{1}(q) f_{2}(q) f_{3}(q) d^{3} q=
$$




$$
\frac{1}{\pi^{4}} \int d^{4} a d^{4} b \int_{x_{0}=t+\lambda_{P}(b-a)_{0}} f_{1}\left(x+\lambda_{P} a\right) f_{2}\left(x+\lambda_{P} b\right) f_{3}(x) \cdot e^{2 i(a, K b)} d^{3} x .
$$

Integrating over the $t$ variable and setting

$$
\beta=2 \lambda_{P}^{-1} K b ; \quad b=\lambda_{P}^{-1} \frac{1}{2} Q \beta,
$$

we get

$$
\int f_{1}(q) f_{2}(q) f_{3}(q) d^{4} q=\frac{1}{(2 \pi)^{2}} \int d^{4} \beta d^{4} x \hat{f}_{1}(\beta) f_{2}\left(x+\frac{1}{2} \lambda_{P}^{-1} Q \beta\right) f_{3}(x) e^{-i \beta x} .
$$

Note that, by the Lebesgue Dominated Convergence Theorem (recall that $f_{i} \in$ $\left.L^{1} \cap \mathcal{F} L^{1}\right)$, the limit of the last expression as $\lambda_{P} \rightarrow 0$ is

$$
\frac{1}{(2 \pi)^{2}} \int d^{4} \beta d^{4} x \hat{f}_{1}(\beta) f_{2}(x) f_{3}(x) e^{i \beta x}=\int d^{4} x f_{1}(x) f_{2}(x) f_{3}(x)
$$

as expected. The correct $\lambda_{P} \rightarrow 0$ limit is also evident in (5.22) or more generally in (5.15).

Note that in (5.22) the non-local corrections to the classical formula are at least quadratic in $\lambda_{P}$ : the linear term vanishes since

$$
\begin{aligned}
\int a_{\mu} e^{2 i(a, K b)} d^{4} a d^{4} b & =-\int b_{\mu} e^{2 i(a, K b)} d^{4} a d^{4} b \\
& =\frac{(2 \pi)^{4}}{2^{4}} \int a_{\mu} \delta^{(4)}(a) d^{4} a=0 .
\end{aligned}
$$

This is a general feature: for each $n, \lambda_{P}$ appears in $\mathcal{D}_{n}$ only through $Q$ (cf. (5.15)) and, in generic units,

$$
Q_{\mu \nu}(\sigma)=\lambda_{P}^{2} \sigma_{\mu \nu} .
$$

Therefore the lowest order corrections to QFT on the usual Minkowski space will be at least quadratic in $\lambda_{P}$. Linear terms in $\lambda_{P}$ might appear only if we introduce gravitational interactions explicitly in the theory.

Finally we discuss the $\mathbb{C}$-valued weight analogous to the classical space integral at fixed time, defined by

$$
\int_{\Sigma^{(l)}} d \sigma \int_{q_{0}=t} f_{1}(q) \ldots f_{n}(q) d^{3} q .
$$

This expression is easily obtained from $(5.14),(5.13)$ evaluating the state $\int_{\Sigma^{(l)}} d \sigma$ over $Z$. A similar comment applies to the explicit formula (5.22) for $n=3$. Note that, for $\sigma \in \Sigma^{(1)}$, we have $K(\sigma)=-\sigma$. For this holds when $\sigma=\sigma_{0},\left(\sigma_{0}^{\mu \nu}\right)=$ $s=\left(\begin{array}{cc}0 & -I \\ I & 0\end{array}\right)$, and $\Sigma^{(1)}$ is a single orbit under the improper rotation group. A standard computation gives

$$
\begin{aligned}
\delta(a, b) & \equiv \int_{\Sigma^{(1)}} d \sigma e^{-2 i a_{\mu} \sigma^{\mu \nu} b_{\nu}} \\
& =-\frac{1}{2}\left(\frac{\sin \gamma_{+}(a, b)}{\gamma_{+}(a, b)}+\frac{\sin \gamma_{-}(a, b)}{\gamma_{-}(a, b)}\right), \\
& \gamma_{ \pm}(a, b)=2\left\|a_{0} \vec{b}-b_{0} \vec{a} \pm \vec{a} \times \vec{b}\right\| .
\end{aligned}
$$

Thus replacing the exponential in $(5.22)$ by $\delta(a, b)$ we get the desired explicit expression for (5.23) in the case $n=3$. 


\section{Towards Quantum Field Theory on the Quantum Spacetime}

In this section we will lay down our basic philosophy and take the first steps towards QFT on QST. A more thorough analysis will be deferred to a subsequent paper.

As required from the outset, the Poincaré group acts as a symmetry group on our QST (cf. Sect. 3 and 4). This fact allows us to retain Wigner's notion of elementary particles as being described by irreducible representations of the covering group of the Poincaré group.

In accordance with one of our principles, the large scale limit of our QST agrees with its classical limit and yields the classical Minkowski space times an unobserved ghost manifold. This asymptotic behaviour is expected to allow one to describe causality for QFT over QST as an asymptotic property corresponding to the locality principle [17] in the large scale limit.

The usual construction of asymptotic scattering states, which also involves a large scale limit, should likewise carry over.

When attempting a perturbative study of the $S$-matrix for QFT over QST, the first steps are to define free fields and interaction Hamiltonians.

Starting from Wigner's definition of particles, the usual Fock space construction yields a free field associated with an irreducible representation of the covering group of the Poincaré group.

There is no difficulty in evaluating this field on the QST, at least formally, as a function with values in the algebra spanned by the creation and destruction operators. For simplicity we consider a neutral scalar free field $\phi(x)$. Evaluating on $q_{\mu}$ according to the rule (5.1) gives

$$
\phi(q)=\frac{1}{(2 \pi)^{3 / 2}} \int\left(e^{i q_{\mu} k^{\mu}} \otimes a(\vec{k})+e^{-i q_{\mu} k^{\mu}} \otimes a(\vec{k})^{*}\right) d \Omega_{m}^{+}(\vec{k}),
$$

where $d \Omega_{m}^{+}(\vec{k})=\frac{d^{3} \vec{k}}{2 \sqrt{\vec{k}^{2}+m^{2}}}$ is the usual invariant measure over the positive energy hyperboloid of mass $m$ :

$$
\Omega_{m}^{+}=\left\{k \in \mathbb{R}^{4} / k_{\mu} k^{\mu}=m^{2}, \quad k_{0}>0\right\} .
$$

In order to give a precise mathematical meaning to the formal expression (6.1) we may think of a quantum field over QST acting on a Hilbert space $\mathcal{H}$ as a linear map, continuous in the appropriate topology, assigning to test functions $f$ linear operators affiliated to the $C^{*}$-tensor product $\mathcal{E} \otimes \mathcal{B}(\mathcal{H})$ and formally denoted by

$$
f \rightarrow \int \phi(q+a I) f(a) d^{4} a
$$

It would be possible, and perhaps even natural, to define a different notion of free field over QST, by letting $\phi$ depend of $q$ and $\sigma \in \Sigma$.

This would amount to choosing the creation and annihilation operators $a$ in the CCR algebra over $L^{2}\left(\mathbb{R}^{3} \times \Sigma\right)$, where $\Sigma$ is equipped with the Lorentz invariant measure $d \sigma$ induced by a Haar measure on $L$. Thus

$$
\int_{\Sigma} f(\sigma) d \sigma \equiv \int_{L} f\left(\Lambda \sigma_{0} \Lambda^{T}\right) d \Lambda
$$


where $\sigma_{0}$ is a reference point in $\Sigma$. This approach would be closer in spirit to quantizing wave functions over QST and will be pursued elsewhere.

As a first simpler choice we will ignore here the possible $\sigma$-dependence of free fields.

From the expression (6.1) for our free field over QST and from the definition (5.3) of derivatives, it is clear that the Klein-Gordon equation holds

$$
\left(\square+m^{2}\right) \phi(q)=0
$$

Let $L \rightarrow \mathcal{U}(L)$ be the unitary representation of the Poincaré group over $\mathcal{H}$ defined by the Fock construction and $\alpha_{L} \equiv \operatorname{AdU}(L)$ the induced action on linear operators acting on $\mathcal{H}$. The relativistic covariance of our free field takes the form

$$
\tau_{L} \otimes \alpha_{L}(\phi(q))=\phi(q),
$$

as a consequence of (4.19) and (6.1). In order to define "local obsevables" in this model, we will consider states $\omega$ over the $C^{*}$-algebra $\mathcal{E}$ of QST as the analogues of test functions defining localization data in spacetime. Pure states (with good localization properties) should play a role analogous to points in classical space.

The free field defines a map from states $\omega \in \mathcal{S}(\mathcal{E})$ to operators on $\mathcal{H}$ by

$$
\phi(\omega) \equiv\langle\omega \otimes i d, \phi(q)\rangle, \quad \omega \in \mathcal{S}(\mathcal{E}) .
$$

Introducing the test function $\psi_{\omega}$ associated to $\omega \in \mathcal{S}(\mathcal{E})$ by

$$
\psi_{\omega}(x)=\int e^{-i k_{\mu} x^{\mu}} \omega\left(e^{i k_{\mu} q^{\mu}}\right) \frac{d^{4} k}{(2 \pi)^{4}},
$$

we can write the field operator $\phi$ evaluated at $\omega \in \mathcal{S}(\mathcal{A})$ as the usual free field smeared out with $\psi_{\omega}$ :

$$
\phi(\omega)=\left\langle\phi, \psi_{\omega}\right\rangle=\int \phi(x) \psi_{\omega}(x) d^{4} x .
$$

Relations (6.5), (6.6) allow us to explore the locality properties of the free field over QST. The commutator of two "values" (6.4) of the field takes the form

$$
\left[\phi(\omega), \phi\left(\omega^{\prime}\right)\right]=i \int \Delta(x-y) \psi_{\omega}(x) \psi_{\omega^{\prime}}(y) d^{4} x d^{4} y .
$$

If we specialize $\omega, \omega^{\prime}$ to translates of a given pure state with optimal localization of the form (4.20), say $\omega=\omega_{a}, \omega^{\prime}=\omega_{b}$, we can compute the commutator (6.7) and study its asymptotic properties. In this case, one easily sees that $\psi_{\omega}$, takes the form

$$
\psi_{\omega_{c}}(x)=(2 \pi)^{-2} e^{-\frac{1}{2} \Sigma_{\mu}(x-c)_{\mu}^{2}} .
$$

The computation for the case $m=0$ can be carried out explicitly and exhibits those aspects of causality which are essential in the massive case, too.

We get, in generic units,

$$
\left[\phi\left(\omega_{a}\right), \phi\left(\omega_{b}\right)\right]=
$$




$$
-i \frac{1}{4 \pi\|\vec{a}-\vec{b}\|} \cdot\left(8 \pi \lambda_{P}^{2}\right)^{-1 / 2}\left(e^{-\frac{1}{8 \lambda_{P}^{2}}\left(\|\vec{a}-\vec{b}\|-(a-b)_{0}\right)^{2}}-e^{-\frac{1}{8 \lambda_{P}^{2}}\left(\|\vec{a}-\vec{b}\|+(a-b)_{0}\right)^{2}}\right) I
$$

There are two important features of the expression (6.9). In spacelike directions it falls off like a Gaussian, hence faster than an exponential. If $a, b$ are kept fixed and we look at the limit $\lambda_{P} \rightarrow 0$, the expression (6.9) converges in the sense of distributions to the usual commutator function of the massless free field, as expected.

We next discuss interaction Hamiltonians, briefly. It is important to note beforehand that the free Hamiltonian of a scalar free field of mass $m$ over QST can be written as the space integral of a "quantum density" using the calculus of Sect. 5,

$$
H=\int_{q_{0}=t} \mathcal{H}(q) d^{3} q .
$$

To this end, note that the Hamiltonian of the ordinary neutral scalar free field can be written as

$$
\begin{aligned}
H & =\int_{x_{0}=t} d^{3} x: \frac{1}{2}\left(\frac{\partial \phi}{\partial x_{0}}(x)^{2}+(\vec{\nabla} \phi(x))^{2}+m^{2} \phi(x)^{2}\right): \\
& =\frac{1}{2} \int_{x_{0}=t} d^{3} x:\left(\frac{\partial \phi}{\partial x_{0}}(x)^{2}-\frac{\partial^{2} \phi}{\partial x_{0}^{2}}(x) \phi(x)\right):,
\end{aligned}
$$

where the double dots indicate Wick ordering. Here the Klein Gordon equation and integration by parts have been used. The relation (5.17), applied to the last integral, shows that (6.10) holds.

Consider now a more than bilinear interaction Hamiltonian density

$$
H_{I}(x)=\lambda: \psi_{1}(x) \ldots \psi_{n}(x):,
$$

where $\psi_{1}, \ldots, \psi_{n}$ are free field multiplets and summation over spin and internal degrees of freedom is implicit, $\lambda$ being a matrix of coupling constants.

It is natural to define an interaction Hamiltonian for QFT over QST associated with the interction (6.11) by the integral defined in Sect. 5 of the expression (6.11) evaluated over the variables $q_{\mu}$ :

$$
H_{I}(t) \equiv \int_{\Sigma^{(1)}} d \sigma \int_{q_{0}=t} d^{3} q \lambda: \psi_{1}(q) \ldots \psi_{n}(q):
$$

By the results in Sect. 5 the expression (6.12) can be calculated and agrees with an effective nonlocal Hamiltonian defined by

$$
\begin{gathered}
H_{I}(t)=\int G\left(x_{1}, \ldots, x_{n} ; t\right) \lambda: \psi_{1}\left(x_{1}\right) \ldots \psi_{n}\left(x_{n}\right): d^{4} x_{1} \ldots d^{4} x_{n}, \\
G\left(x_{1}, \ldots, x_{n} ; t\right)=\int_{\Sigma^{(1)}} d \sigma \mathcal{D}_{n}\left(x_{1}, \ldots, x_{n} ; t\right),
\end{gathered}
$$

where the kernel $\mathcal{D}_{n}$ is given by (5.15). In the important case of a trilinear interaction, we get (cf. (5.22))

$$
H_{I}(t)=\frac{1}{\pi^{4}} \int d^{4} a d^{4} b \int_{x_{0}=t+\lambda_{P}(b-a)_{0}} \lambda: \psi_{1}\left(x+\lambda_{P} a\right) \psi_{2}\left(x+\lambda_{P} b\right) \psi_{3}(x): \delta(a, b) d^{3} x,
$$


where the kernel $\delta$ has been calculated in (5.24) and we have used generic units. Leaving out the integration over $d^{3} x$ in Eq. (6.14) we get, of course, the effective nonlocal Hamiltonian density.

Interacting fields can be tentatively defined in the following way: since $\phi$ is a solution of the Klein-Gordon equation, the form

$$
\langle\phi, f\rangle_{t}=\int_{\Sigma} d \sigma \int_{q_{0}=t} d^{3} q\left(\phi(q) \partial_{0} f(q, \sigma)-\partial_{0} \phi(q) f(q, \sigma)\right)
$$

is independent of $t$ for any solution $f \in M(\mathcal{E})$ of the Klein-Gordon equation. If $U(t, s)$ is the unitary evolution operator with

$$
\begin{gathered}
U(t, s) U(s, r)=U(t, r), \\
U(t, t)=1, \\
\frac{d}{d t} U(t, s)=i H_{I}(t) U(t, s),
\end{gathered}
$$

we define the interacting field $\phi_{\text {int }}$ by

$$
\left\langle\phi_{\mathrm{int}}, f\right\rangle_{t}=U(t, 0)\langle\phi, f\rangle_{0} U(0, t) .
$$

Since $\phi_{\text {int }}$ is not a solution of the wave equation, in general, the lefthand side depends on $t$.

By choosing suitable solutions of the wave equation for every $t$ and integrating over $t$ we determine $\phi_{\text {int }}$ on the whole state space of $\mathcal{E}$.

Finally, we may follow the usual procedure and postulate LSZ asymptotic conditions which then lead to the ordinary perturbation expansion of the $S$-matrix.

As usual the $n^{\text {th }}$ order contribution can be calculated from the $n^{\text {th }}$ order contribution to the time-ordered function obtained substituting $H_{I}(t)$ in the formula

$$
\frac{i^{n}}{n !} \int d t_{1} \ldots d t_{n}\left(\Omega, T\left(A\left(x_{1}\right) \ldots A\left(x_{m}\right) H_{I}\left(t_{1}\right) \ldots H_{I}\left(t_{n}\right)\right) \Omega\right)
$$

where the ordering now refers to the $x_{1_{0}}, \ldots, x_{m_{0}}, t_{1}, \ldots, t_{n}$ variables rather than to the integration variables in (6.13). This modifies the usual Feynmann rules. Due to the non-local character of our effective interaction, for renormalizable theories, the renormalized perturbative expansion should agree with the classical one for $\lambda_{P}=0$ and contain corrections of order $\lambda_{P}^{2}$ due to the quantum nature of spacetime. We intend to give a more thorough discussion of these points elsewhere. 


\section{Outlook}

The choice of our QST was motivated by our principle that spacetime should have an operational meaning; we observed that this meaning would be destroyed by the gravitational collapse caused by preparing a very sharply localized state.

We wish to point out that our uncertainty relations (2.8) and (2.9) appear as necessary but not a priori sufficient conditions to prevent gravitational collapse in such a process. Hence the Quantum Structure of our spacetime might reflect only part of the necessary restrictions.

The very first steps to QFT over QST outlined in Sect. 6 show that the quantum structure of our model of spacetime does lead to a smoothing of ultraviolet divergences. A more thorough analysis of the surviving divergences and their renormalization in specific models is required (see [23] for related results).

Properties of the resulting theory which can be tested at a perturbative level (cluster properties, convergence of asymptotic states) can be a guide to explore properties of QFT over QST.

We wish to point out several other open problems.

Causality in QST has been mentioned here as an asymptotic property in the limit of vanishing Planck length. However, there might be an exact form, related to the causality properties of the free field over QST, expressing the propagation of wave functions over QST. But even if such a tighter condition existed, it is not obvious whether we can expect any stability under interactions.

We note that the $\Sigma$-dependence of interactions has been removed by a procedure which is not Lorentz invariant. Other approaches are possible, of course, e.g. one could treat the variables $\sigma \in \Sigma$ as random variables. Or one could adopt a dynamical treatment introducing $\sigma$-dependent fields. We believe that a satisfactory understanding of the role of $\Sigma$ should be possible if one were to incorporate gravitation in the theory. Our QST ought to offer a more suitable basis for the formulation of Quantum Gravity, as it embodies part of the limitations on the structure of spacetime determined by gravity, at least at a semiclassical level.

More generally, we could use the gauge principle as a natural way of introducing interactions between fields over the QST and Connes' non-commutative geometry [8] should provide the right framework here. The quantum nature of spacetime has the effect of making QED into a non-commutative gauge theory, so that even the theory of electro-magnetic fields without matter should become an interacting theory.

The large scale structure of the univese and the microstructure of spacetime relate to quite distinct asymptotic regimes. Yet it would be interesting and instructive to generalize our quantization procedure to a general curved spacetime. The methods of deformation and geometric quantization could well apply here [24-26]. In this context, it is quite clear that our formalism is not at all intended to exclude black hole formation at scales which are larger than the Planck length.

Finally, it would be important to investigate whether a version of the Euclidean approach to QFT is possible for QFT over QST.

We hope to come back to these and related questions in a continuation of the present paper. 


\section{Appendix A}

Let $\mathfrak{A}$ be a $C^{*}$-algebra with unit I. A selfajdoint operator $A$ affiliated to $\mathfrak{A}$ is defined by a $*$-homomorphism (with unbounded support if $A$ is unbounded) of $\mathcal{C}_{0}(\mathbb{R})$ into $\mathfrak{A}$, denoted

$$
f \in \mathcal{C}_{0}(\mathbb{R}) \rightarrow f(A) \in \mathfrak{A},
$$

whose support projection $E \in \mathfrak{A}^{* *}$ is central. Equivalently, if $\left\{f_{n} ; n=0,1,2, \ldots,\right\} \subset$ $\mathcal{C}_{0}(\mathbb{R})_{+}$is a sequence in the unit ball converging pointwise to $I$, then, for each $B \in \mathfrak{A}$,

$$
\left\|\left[f_{n}(A), B\right]\right\|_{n \rightarrow \infty} \rightarrow 0 .
$$

We will refer to $E$ as the support of definition of $A$ (or just support, if no confusion is possible).

If $\mathfrak{A}$ has no unit, $A$ is said to be affiliated to $\mathfrak{A}$ if it is affiliated to the multiplier algebra $M(\mathfrak{A})$.

A state $\omega$ (a representation $\pi$ ) of $\mathfrak{A}$ is in the support of $A$ if $\tilde{\omega}(E)=1$ (resp. $\tilde{\pi}(E)=I)$, where $\tilde{\omega}, \tilde{\pi}$ denote the normal extensions to $\mathfrak{A}^{* *}$.

The normal extension of $f \rightarrow f(A) \in \mathfrak{A}$ to bounded Borel functions $f$ on $\mathbb{R}$ will be also denoted $f \in \mathcal{B}(\mathbb{R}) \rightarrow f(A) \in \mathfrak{A}^{* *}$. If $E(\lambda) \equiv \chi_{(-\infty, \lambda]}(A), \lambda \in \mathbb{R}, E$ is the strong limit of $E(\lambda)$ as $\lambda \rightarrow \infty$ and $\lambda \in \mathbb{R} \rightarrow \tilde{\pi}(E(\lambda))$ is a spectral family for each $\pi$ in the support of $A$. For such $\pi, \pi(A)$ will denote the selfadjoint operator with spectral resolution $\tilde{\pi}(E(\lambda))$.

We will say that the selfadjoint operator $A$ affiliated to $\mathfrak{A}$ is central if $f(A)$ is in the centre of $\mathfrak{A}$ (or of $M(\mathfrak{A})$, if $\mathfrak{A}$ has no unit) for each $f \in \mathcal{C}_{0}(\mathbb{R})$, i.e. if, for each representation in the doman of $A$,

$$
\pi(A) \eta \pi(\mathfrak{A})^{\prime} \cap \pi(\mathfrak{A})^{\prime \prime} .
$$

Since $\pi(A)$ is anyway affiliated to $\pi(\mathfrak{A})^{\prime \prime}, \pi(A) \eta \pi(\mathfrak{A})^{\prime}$ would suffice.

A state $\omega \in \mathcal{S}(\mathfrak{A})$ will be said to be in the domain of $A$ if it is in the support of $A$ and

$$
\sup \left\{\omega(f(A)) ; f \in \mathcal{C}_{0}(\mathbb{R})_{+}, \quad f(\lambda) \leq \lambda^{2}, \lambda \in \mathbb{R}\right\} \equiv \omega\left(A^{2}\right)<\infty .
$$

In this case we may and will write

$$
\omega(A)=\int_{-\infty}^{+\infty} \lambda d \tilde{\omega}(E(\lambda))
$$

We can now state some easy facts.

I. A state $\omega \in \mathcal{S}(\mathfrak{A})$ is in the support of definition of $A$ if and only if if the same applies to the GNS representation $\pi_{\omega}$.

Furthermore, $\omega$ is in the domain of $A$ if and only if $\xi_{\omega}$ is in the domain of $\pi_{\omega}(A)$, in which case we have

$$
\omega(A)=\left(\xi_{\omega}, \pi_{\omega}(A) \xi_{\omega}\right) .
$$

If $\omega$ is in the domain of $A$ we may define

$$
\left(\Delta_{\omega} A\right)^{2}=\omega\left(A^{2}\right)-\omega(A)^{2}=\left\|\left(\pi_{\omega}(A)-\omega(A) I\right) \xi_{\omega}\right\|^{2} .
$$


Of course we will say that $\omega$ is definite on $A$ if $\omega\left(A^{2}\right)=\omega(A)^{2}$, i.e. if $\pi_{\omega}(A) \xi_{\omega}=$ $\omega(A) \xi_{\omega}$.

II. Let $\nu$ be a regular probability measure on $\mathcal{S}(\mathfrak{A})$ with barycentre $\omega \in \mathcal{S}(\mathfrak{A})$. Then $\omega$ is in the support of $A$ iff $\nu$ is carried by the states in the support of $A$.

Moreover, $\omega$ is in the domain of $A$ if and only if $\nu$ is carried by the states in the domain of $A$ and $\varphi \rightarrow \varphi\left(A^{2}\right)$ is $\nu$-integrable. This implies that $\varphi \rightarrow \varphi(A)$ is $\nu$-square integrable since $\varphi(A)^{2} \leq \varphi\left(A^{2}\right)$, hence $\varphi \rightarrow \varphi(A)$ is integrable and $\varphi \rightarrow \Delta_{\varphi} A$ is square integrable.

If $\omega$ is also in the domain of another selfadjoint operator $B$ affiliated to $\mathfrak{A}$, we have as in Proposition 3.3,

$$
\begin{aligned}
\Delta_{\omega} A & \geq \int \Delta_{\varphi} A d \nu(\phi), \\
\Delta_{\omega} A \Delta_{\omega} B & \geq \int \Delta_{\varphi} A \Delta_{\varphi} B d \nu(\varphi) .
\end{aligned}
$$

III. If $\alpha$ is an automorphism of $\mathfrak{A}$ and $A$ a selfadjoint operator affiliated to $\mathfrak{A}$, we can define $\alpha(A)$ affiliated to $\mathfrak{A}$ by the homomorphism

$$
f \in \mathcal{C}_{0}(\mathbb{R}) \rightarrow f(\alpha(A)) \in M(\mathfrak{A})
$$

s.t. $f(\alpha(A))=\tilde{\alpha}(f(A))$, where $\tilde{\alpha} \in$ Aut $M(\mathfrak{A})$ is the extension of $\alpha$ defined by

$$
\alpha(B C)=\tilde{\alpha}(B) \alpha(C), \quad B \in M(\mathfrak{A}), \quad C \in \mathfrak{A} .
$$

IV. If $A_{1}, \ldots, A_{n}$ are selfadjoint operators affiliated to $\mathfrak{A}$, we say that they commute if the $f_{i}\left(A_{i}\right)$ commute with one another for each $f_{i} \in \mathcal{C}_{0}(\mathbb{R}), i=1,2, \ldots, n$. In this case, the map $f_{1} \otimes \cdots \otimes f_{n} \in \mathcal{C}_{0}\left(\mathbb{R}^{n}\right) \rightarrow f_{1}\left(A_{1}\right) \ldots f_{n}\left(A_{n}\right) \in M(\mathfrak{A})$ extends to a $*$-homomorphisms $\mathcal{C}_{0}\left(\mathbb{R}^{n}\right)$ into $M(\mathfrak{A})$ whose support is called the joint spectrum of $A_{1}, \ldots, A_{n}$.

V. If $A$ is a selfadjoint operator affiliated to a $C^{*}$-algebra $\mathfrak{A}$ and $\omega$ is a state on $\mathfrak{A}$ in the domain of $A$, the state of $\mathfrak{A}$ induced by the vector $\omega\left(A^{2}\right)^{-1 / 2} \pi_{\omega}(A) \xi_{\omega}$ in the representation $\pi_{\omega}$ will be denoted by $\omega_{A}$. If $A, B$ are selfadjoint operators affiliated to $\mathfrak{A}$, we will say that $\omega$ is in the domain of their commutator if $\omega_{A}$ is in the domain of $B$ and $\omega_{B}$ in the domain of $A$, i.e. if $\xi_{\omega}$ is in the domain of $\left[\pi_{\omega}(A), \pi_{\omega}(B)\right]$. If $A$, $B, C$ are selfadjoint operators affiliated to $\mathfrak{A}$ with the same support $E$ such that, for each representation $\pi$ in that support $(\tilde{\pi}(E)=I)$, the commutator $[\pi(A), \pi(B)]$ has closure $i \pi(C)$, we will write

$$
[A, B]^{-}=i C
$$




\section{Appendix B}

I. The manifold $\Sigma_{+}$is homeomorphic to $T S^{2}$, the tangent bundle to the twodimensional sphere.

Let $\{\vec{n}, \vec{v}\}$ denote the generic point in $T S^{2}$, where $\vec{n} \in S^{2}$ is a unit vector in $\mathbb{R}^{3}$ and $\vec{v} \in \mathbb{R}^{3}$ is s.t. $\vec{v} \cdot \vec{n}=0$.

For each $\sigma \in \Sigma_{+}$let $\sigma=(\vec{e}, \vec{m})$ be the parametrization by electric and magnetic components, so that $\vec{e}^{2}=\vec{m}^{2}, \vec{e} \cdot \vec{m}=1$. Hence $\vec{e}+\vec{m} \neq 0$ and we can define

$$
\vec{n}_{\sigma} \equiv\|\vec{e}+\vec{m}\|^{-1}(\vec{e}+\vec{m}) .
$$

If $\sigma \in \Sigma_{+}, \sigma=(\vec{e}, \vec{m})$ with $\vec{e}=\vec{m}$, let $h(\sigma) \in T S^{2}$ be defined by

$$
h(\sigma)=\left\{\vec{n}_{\sigma}, \overrightarrow{0}\right\}=\{\vec{e}, \overrightarrow{0}\}=\{\vec{m}, \overrightarrow{0}\} .
$$

If $\vec{e} \neq \vec{m}$, let $\vec{u}_{\sigma}=\|\vec{e} \times \vec{m}\|^{-1} \vec{e} \times \vec{m}$, and let $L_{\sigma}$ be the boost along $\vec{u}_{\sigma}$ with speed $\beta>0$ s.t.

$$
\vec{e}^{2}=\vec{m}^{2}=\frac{1+\beta^{2}}{1-\beta^{2}} .
$$

Then $L_{\sigma}$ takes $\sigma^{\prime}=\left(\vec{n}_{\sigma}, \vec{n}_{\sigma}\right) \in \Sigma_{+}$to $\sigma=(\vec{e}, \vec{m}) \in \Sigma_{+}$:

$$
L_{\sigma} \sigma^{\prime} L_{\sigma}^{T}=\sigma
$$

Choose $\chi \in[0,+\infty)$ s.t. $\vec{e}^{2}=\vec{m}^{2}=\cosh 4 \chi$, i.e. $\gamma=\left(1-\beta^{2}\right)^{-1 / 2}=\cosh 2 \chi$. We can define a homeomorphism $h: \sigma \in \Sigma_{+} \rightarrow h(\sigma) \in T S^{2}$ by setting

$$
h(\sigma)=\left\{\vec{n}_{\sigma}, \vec{v}_{\sigma}\right\} ; \quad \vec{v}_{\sigma}=\chi \vec{u}_{\sigma} .
$$

II. For each point $\vec{n}_{\sigma} \in S^{2}$ there is a Borel section for the action of $L_{+}^{\uparrow}$ on $\Sigma_{+}$:

$$
\begin{gathered}
\sigma \in \Sigma_{+} \rightarrow \Lambda_{\sigma} \in L_{+}^{\uparrow}, \\
\Lambda_{\sigma} \sigma_{0} \Lambda_{\sigma}^{T}=\sigma, \quad \sigma \in \Sigma_{+},
\end{gathered}
$$

where $\sigma_{0}=\left(\vec{e}_{0}, \vec{e}_{0}\right), \vec{e}_{0}=(0,1,0)$, s.t.

$$
\begin{gathered}
\Lambda_{\sigma}=L_{\sigma} R_{\sigma} ; \quad L_{\sigma} \text { a boost, } R_{\sigma} \text { a rotation, } \\
\sigma \rightarrow L_{\sigma} \quad \text { is continuous on } \Sigma_{+}, \\
\sigma \in \Sigma_{+} \backslash\left\{\sigma / \vec{n}_{\sigma}=\vec{n}_{0}\right\} \rightarrow R_{\sigma} \text { is continuous. }
\end{gathered}
$$

We let $L_{\sigma}$ be defined as in I; it suffices to choose a continuous map $\vec{n} \in S^{2} \backslash\left\{\vec{n}_{0}\right\} \rightarrow$ $R(\vec{n})$ from the 2 -sphere minus one point to the rotation group such that $R(\vec{n}) \vec{e}_{0}=\vec{n}$. We can take $R\left(\vec{n}_{0}\right)$ to be an arbitrary rotation taking $\vec{e}_{0}$ to $\vec{n}_{0}$ and set

$$
R_{\sigma}=R\left(\vec{n}_{\sigma}\right) .
$$

If $\vec{n}_{0} \neq-\vec{e}_{0}$, the map $R(\vec{n})$ can be defined as the identity if $\vec{n}=\vec{e}_{0}$ or as the rotation around $\vec{e}_{0} \times \vec{n}$ which takes $\vec{e}_{0}$ to $\vec{n}$ if $\vec{n} \neq \vec{e}_{0}$. If $\vec{n}_{0}$ is arbitrary, replacing $\vec{e}_{0}$ by $-\vec{n}_{0}$ 
in the above construction yields a map $R^{\prime}(\vec{n})$ and, for any fixed choice of a rotation $R$ which takes $\vec{e}$ to $\vec{n}_{0}, R(\vec{n}) \equiv R^{\prime}(\vec{n}) R$ meets the desired requirements.

If $P$ denotes the space reflection, $\sigma_{P}=(-\vec{e}, \vec{m}) \in \Sigma_{\mp}$ if $\sigma=(\vec{e}, \vec{m}) \in \Sigma_{ \pm}$; we can define $\Lambda_{\sigma} \equiv P L_{\sigma_{P}} R_{\sigma_{P}}, \sigma \in \Sigma$, thus extending our section to $\Sigma$ and letting it take values in the full Lorentz group.

Finally we point out yet another picture of $\Sigma$ which might turn out to be useful. Associate to $\sigma \in \Sigma, \sigma=(\vec{e}, \vec{m})$, the vector $\vec{u}=\vec{e}+i \vec{m} \in \mathbb{C}^{3}$. Then $v^{2}=\Sigma_{j} v_{j}^{2}=$ $e^{2}-m^{2}+2 i \vec{e} \cdot \vec{m}$, so that the image of $\Sigma$ is the manifold $\left\{v \in \mathbb{C}^{3} / v^{2}= \pm 2 i\right\}$. The

action of $L_{+}^{\uparrow}$ on $\Sigma$ corresponds to the action of $\mathcal{O}(3, \mathbb{C})$ on that manifold, the space or time reflection to complex conjugation, and the Euclidean norm $\|\sigma\|, \sigma \in \Sigma$ to the norm $\|v\|=\frac{1}{\sqrt{2}}(v, v)^{1 / 2}=\left(\frac{1}{2} \Sigma_{j}\left|v_{j}\right|^{2}\right)^{1 / 2}$.

\section{Appendix $\mathbf{C}$}

The goal of this appendix is to calculate the kernel $\mathcal{D}_{n}$ and we begin by calculating the kernels describing the multiplication in $\mathcal{E}$.

Let $f(q)=\int d^{4} k \hat{f}(k) e^{i k q}$. The function $f(x)=\int d^{4} k \hat{f}(k) e^{i k x}$ is called the symbol of $f(q)$. We formulate the multiplication in $\mathcal{E}$ in terms of these symbols. The symbol of a product is

$$
\left(f_{1} \ldots f_{n}\right)(x)=\int d^{4} x_{1} \ldots d^{4} x_{n} f_{1}\left(x_{1}\right) \ldots f_{n}\left(x_{n}\right) C_{n}\left(x_{1}-x, \ldots, x_{n}-x\right),
$$

where the distribution $C_{n}$ is given by

$$
C_{n}\left(x_{1}, \ldots, x_{n}\right)=(2 \pi)^{-4} \int d^{4} k_{1} \ldots d^{4} k_{n} e^{-i \Sigma k_{j} x_{j}+\frac{i}{2} \Sigma_{j<l} k_{j} Q k_{l}} .
$$

The quadratic form in the exponential can be written in the form

$$
\sum_{j<l} k_{j} Q k_{l}=\frac{1}{2}(\underline{k}, B \otimes Q \underline{k}),
$$

where $\underline{k}=\left(k_{1}, \ldots, k_{n}\right) \in \mathbb{R}^{n} \otimes \mathbb{R}^{4}$ and $B$ is an $n \times n$-matrix with entries $B_{j l}=1$, $j<l, B_{j l}=0, j=l, B_{j l}=-1, j<l$. If $n$ is even, the matrix $B$ has an inverse $B^{-1}$, with entries $\left(B^{-1}\right)_{j l}=(-1)^{j+l} B_{j l}$. Hence in this case we obtain

$$
C_{n}\left(x_{1}, \ldots, x_{n}\right)=\pi^{-2 n} e^{-2 i \Sigma_{j<l}(-1)^{j+l} x_{j} Q^{-l} x_{l}}, \quad n \text { even, }
$$

where we have used the fact that $B$ and $Q$ have determinant one.

For later convenience we introduce coordinates $y_{j}=\sum_{l=1}^{j}(-1)^{l} x_{l}$. In these coordinates the quadratic form in the exponential assumes the simple form

$$
\sum_{j<l}(-1)^{j+l} x_{j} Q^{-1} x_{l}=\sum_{j=1}^{n-1} y_{j} Q^{-1} y_{j+1}
$$

If $n$ is odd the matrix $B$ is not invertible. In this case we set $f_{1}, \ldots, f_{n}=$ $f_{1}, \ldots, f_{n} f_{n+1}$ with $f_{n+1}=1$. 
Since the symbol of the unit is the function $f_{n+1}(x)=1$ we obtain $C_{n}$ from $C_{n+1}$ by integrating over the last variable. Hence

$$
\begin{aligned}
C_{n}\left(x_{1}, \ldots, x_{n}\right) & =\int d^{4} x_{n+1} C_{n+1}\left(x_{1}, \ldots, x_{n+1}\right) \\
& =\pi^{-2(n+1)} e^{-2 i \Sigma_{j=1}^{n-1} y_{j} Q^{-1} y_{j+1}} \int d^{4} y_{n+1} e^{-2 i y_{n} Q^{-1} y_{n+1}} \\
& =\pi^{-2(n+1)} e^{-2 i \Sigma_{j=1}^{n-1} y_{j} Q^{-1} y_{j+1}}(2 \pi)^{4} \delta^{(4)}\left(2 Q^{-1} y_{n}\right) \\
& =\pi^{-2(n-1)} e^{-2 i \Sigma_{j=1}^{n-2} y_{j} Q^{-1} y_{j+1}} \delta^{(4)}\left(y_{n}\right) \\
& =C_{n-1}\left(x_{1}, \ldots, x_{n-1}\right) \delta^{(4)}\left(\sum_{j=1}^{n}(-1)^{j} x_{j}\right), n \text { odd } .
\end{aligned}
$$

If we replace $x_{j}$ by $x_{j}-x$ the coordinates $y_{j}$ with $j$ even remain unchanged whereas for $j$ odd $y_{j}$ is replaced by $y_{j}+x$. We have

$$
\sum_{j=1}^{2 n} y_{j} Q^{-1} y_{j+1}=\sum_{j=1}^{n} y_{2 j} Q^{-1}\left(y_{2 j+1}-y_{2 j-1}\right),
$$

hence this expression will remain invariant.

Therefore we find

$$
C_{2 n}\left(x_{1}-x, \ldots, x_{2 n}-x\right)=\pi^{-4 n} e^{-2 i \Sigma_{j=1}^{2 n-1} y_{j} Q^{-1} y_{j+1}} e^{-2 i x Q^{-1} y_{2 n}}
$$

and

$$
\begin{aligned}
C_{2 n+1}\left(x_{1}-x, \ldots, x_{2 n+1}-x\right) & =\pi^{-4 n} e^{-2 i \Sigma_{j=1}^{2 n-1} y_{j} Q^{-1} y_{j+1}} e^{-2 i x Q^{-1} y_{2 n}} \delta^{(4)}\left(y_{2 n+1}+x\right) \\
& =\pi^{-4} e^{-2 i \Sigma_{j=1}^{2 n} y_{j} Q^{-1} y_{j+1}} \delta^{(4)}\left(y_{2 n+1}+x\right) . \quad(\text { C.9) }
\end{aligned}
$$

The integral over a spatial hyperplane $x^{0}=t$ can now be easily performed. We obtain

$$
\int_{x^{0}=t} d^{3} x C_{2 n+1}\left(x_{1}-x, \ldots, x_{2 n+1}-x\right)=\pi^{-4 n} e^{-2 i \Sigma_{j=1}^{2 n} y_{j} Q^{-1} y_{j+1}} \delta\left(y_{2 n+1}^{0}+t\right) \quad(C .10)
$$

in the odd case and

$$
\begin{aligned}
\int_{x^{0}=t} & d^{3} x C_{2 n+2}\left(x_{1}-x, \ldots, x_{2 n+2}-x\right) \\
& =\frac{1}{2 \pi} \int d \eta \int d^{4} x e^{i \eta\left(x^{0}-t\right)} C_{2 n+2}\left(x_{1}-x, \ldots, x_{2 n+2}-x\right) \\
& =\frac{1}{2 \pi} \int d \eta \int d^{4} x \pi^{-4(n+1)} e^{-2 i \Sigma_{j=1}^{2 n+1} y_{j} Q^{-1} y_{j+1}} e^{-2 i x Q^{-1}\left(y_{2 n+2}-\frac{1}{2} Q(\eta, \overrightarrow{0})\right)} e^{-i \eta t} \\
& =\frac{1}{2 \pi} \int d \eta \pi^{-4} e^{-2 i \Sigma_{j=1}^{2 n} y_{j+1}} e^{-i\left(y_{2 n+1}^{0}+t\right)} \delta^{(4)}\left(y_{2 n+2}-\frac{1}{2} Q(\eta, \overrightarrow{0})\right)
\end{aligned}
$$

in the even case. Using (C.7) and the formulas

$$
y_{2 j}=\sum_{l=1}^{j}\left(x_{2 l}-x_{2 l-1}\right), \quad y_{2 j+1}-y_{2 j-1}=x_{2 j}-x_{2 j+1},
$$




$$
y_{2 n+1}^{0}+t=\sum_{j=1}^{2 n+1}(-1)^{j}\left(x_{j}^{0}-t\right)
$$

we obtain the kernels

$$
\mathcal{D}_{n}\left(x_{1}, \ldots, x_{n} ; t\right)=\int_{x^{0}=t} d^{3} x C_{n}\left(x_{1}-x, \ldots, x_{n}-x\right)
$$

in the form

$$
\mathcal{D}_{2 n+1}\left(x_{1}, \ldots, x_{2 n+1} ; t\right)=\pi^{-4 n} e^{2 i A_{n}\left(x_{1}-x_{2}, \ldots, x_{2 n}-x_{2 n+1}\right)} \cdot \delta\left(\sum_{j=1}^{2 n+1}(-1)^{j}\left(x_{j}^{0}-t\right)\right)
$$

and

$$
\begin{gathered}
\mathcal{D}_{2 n+2}\left(x_{1}, \ldots, x_{2 n+2} ; t\right)=\pi^{-4 n} e^{2 i A_{n}\left(x_{1}-x_{2}, \ldots, x_{2 n}-x_{2 n+1}\right)} \\
\frac{1}{2 \pi} \int d \eta e^{i \eta \Sigma_{j=1}^{2 n+1}(-1)^{j}\left(x_{j}^{0}-t\right)} \delta^{(4)}\left(\sum_{j=1}^{n+1}\left(x_{2 j}-x_{2 j-1}\right)-\frac{1}{2} Q(\eta, \overrightarrow{0})\right),
\end{gathered}
$$

where $A_{n}$ is the quadratic form on $\mathbb{R}^{2 n} \times \mathbb{R}^{4}$ given by

$$
A_{n}\left(u_{1}, \ldots, u_{2 n}\right)=\sum_{j \leq l \leq n} u_{2 j-1} Q^{-1} u_{2 l}
$$

Acknowledgements. It is our pleasure to thank D. Buchholz, A. Connes and R. Haag for discussions.

We gratefully acknowledge the support given by the Graduiertenkolleg "Theoretische Elementarteilchenphysik" for a visit of S.D. to Hamburg and by the CNR and the Dipartimento di Matematica, University of Rome I for visits of K.F. to Rome thus making our collaboration possible.

S.D. takes pleasure in thanking Colin Sutherland for the warm hospitality and ideal working conditions offered to him at the Department of Pure Mathematics, University of New South Wales, in a stage of this research.

Note added in proof: The model of QST proposed here is the simplest but not the unique one implementing our Uncertainty Relations (2.8), (2.9). In other models with this property the commutators of the q's are no longer central, the associated algebras admit representations where the stronger uncertainty relations deduced in Section 2 are satisfied, namely $\inf \left(\Delta q_{j} ; j=1,2,3\right) \cdot \sup \left(\Delta q_{k}, k=1,2,3\right) \gtrsim 1$ holds too in these representations, however, translation invariance at the Planck scale is spontaneously broken [27].

We are grateful to A. Kempf for pointing out to us references $[28,29]$. 


\section{References}

1. Amati, D., Ciafaloni, M., Veneziano, G.: Nucl. Phys. B347, 551 (1990); Amati, D.: On Spacetime at Small Distances. In: Sakharov Memorial Lectures, Kladysh, L.V., Fainberg, V.Ya., eds., Nova Science P. Inc., 1992

2. Ellis, J., Mavromatos, N.E., Nanopoulos, D.V.: A Liouville String Approach to Microscopic Time in Cosmology. Preprint CERN-TH. 7000/93

3. Ashtekar, A.: Quantum Gravity: A Mathematical Physics Perspective. Preprint CGPG-93/12-2

4. Kempf, A.: Uncertainty Relations in Quantum Mechanics with Quantum Group Symmetry. Preprint DAMT/93-65

5. Woronowicz, S.L.: Compact Matrix Pseudogroups. Commun. Math. Phys. 111, 613-665 (1987)

6. Wheeler, J.A.: Geometrodynamics and the Issue of the Final State. In: Relativity, Groups and Topology. De Witt, C., De Witt, B., (eds.) Gordon and Breach 1965. Hawking, S.W., Spacetime Foam. Nucl. Phys. B144, 349, (1978)

7. Madore, J.: Fuzzy Physics. Ann. Phys. 219, 187-198 (1992)

8. Connes, A.: Non-Commutative Geometry. Academic Press, 1994

9. Connes, A., Lott, J.: Particle Models and Non Commutative Geometry. Nucl. Phys. B. (Proc. Suppl.) 11B, 19-47 (1990); Kastler, D.: A detailed account of Alain Connes' version of the standard model in non-commutative geometry, I, II. Rev. Math. Phys. 5, 477-532 (1993); III, Preprint CPT-92/P. 2814; IV (with Th. Schücker), Preprint CPT-94/P.3092

10. Schrader, R.: The Maxwell Group and the Quantum Theory of Particles in Classical Homogeneous Elecric Fields. Forts. der Phys. 20, 701-734 (1972)

11. Filk, T.: Field Theory on the Quantum Plane. Preprint, University of Freiburg, August 1990, THEP 90/12

12. Grosse, H., Madore, J.: A Noncommutative Version of the Schwinger Model. Phys. Lett. B283, 218 (1992)

13. Mack, G., Schomerus, V.: Models of Quantum Space Time: Quantum Field Planes. Preprint, Harvard University HUTMP 93-B335. Lukierski, J., Ruegg, H.: Quantum $\kappa-$ Poincaré in any Dimension. Phys. Lett. B, to appear

14. Doplicher, S., Fredenhagen, K., Roberts, J.E.: Spacetime Quantization Induced by Classical Gravity. Phys. Lett. B 331, 33-44 (1994)

15. Hawking, S.W., Ellis, G.F.R.: The large scale structure of spacetime. Cambridge: Cambridge U.P., 1973

16. Knight, J.M.: Strict localization in Quantum Field Theory. J. Math. Phys. 2, 439-471 (1961); Licht, A.L.: Strict localization. J. Math. Phys. 4, 1443 (1963)

17. Haag, R.: Local Quantum Physics. Berlin, Heidelberg, New York: Springer TMP, 1993

18. Straumann, N.: General Relativity and Relativistic Astrophysics. Berlin-Heidelberg-New York: Springer, 1984

19. Woronowicz, S.L.: Unbounded elements affiliated with $C^{*}$-algebras and noncompact Quantum Groups. Commun. Math. Phys. 136, 399-432 (1991). Woronowicz, S.L.: $C^{*}$-Algebras Generated by Unbounded Elements. Preprint 1994; Baaj, S.: Multiplicateur non borné. Thèse, Université Paris VII 1980

20. Pedersen, G.K.: $C^{*}$-Algebras and their Automorphism Groups. New York: Acad. Press, 1979

21. Dixmier, J.: $C^{*}$-Algebras. Amsterdam: North Holland, 1980 
22. Von Neumann, J.: Über die Eindeutigkeit der Schrödingerschen Operatoren. Math. Annalen 104, 570-578 (1931)

23. Filk, T.: Renormalizability of Field Theories on Quantum Spaces, Preprint University of Freiburg, THEP 94/15, 1994

24. Didonato P. et al. eds.: Sympletic Geometry and Mathematical Physics. Actes du Colloque en Honneur de J.M. Souriau, Basel, Boston: Birkhäuser, 1991

25. Rieffel, M.A.: Deformation Quantization of Heisenberg Manifolds. Commun. Math. Phys. 122, 531-562 (1989)

26. Landsman, N.P.: Strict Deformation Quantization of a Particle in External Gravitational and Yang Mills Fields. J. Geom. Phys. 12, 93-132 (1993)

27. Doplicher, S., Fredenhagen, K.: in preparation

28. Maggiore, M.: Quantum Groups, Gravity, and the generalized uncertainty principle, Phys. Rev. D49, 5182-5187 (1994); Phys. Lett. B 304, 65-69 and 319, 83-86 (1993)

29. Garay, L.J.: Quantum Gravity and minimum Length. Preprint Imperial/TP/9394/20, gr-qc/9403008 